\title{
DISCONTINUOUS FINITE ELEMENT METHODS FOR A BI-WAVE EQUATION MODELING $d$-WAVE SUPERCONDUCTORS
}

\author{
XIAOBING FENG AND MICHAEL NEILAN
}

\begin{abstract}
This paper concerns discontinuous finite element approximations of a fourth-order bi-wave equation arising as a simplified Ginzburg-Landautype model for $d$-wave superconductors in the absence of an applied magnetic field. In the first half of the paper, we construct a variant of the Morley finite element method, which was originally developed for approximating the fourthorder biharmonic equation, for the bi-wave equation. It is proved that, unlike the biharmonic equation, it is necessary to impose a mesh constraint and to include certain penalty terms in the method to guarantee convergence. Nearly optimal order (off by a factor $|\ln h|$ ) error estimates in the energy norm and in the $H^{1}$-norm are established for the proposed Morley-type nonconforming method. In the second half of the paper, we develop a symmetric interior penalty discontinuous Galerkin method for the bi-wave equation using general meshes and prove optimal order error estimates in the energy norm. Finally, numerical experiments are provided to gauge the efficiency of the proposed methods and to validate the theoretical error bounds.
\end{abstract}

\section{INTRODUCTION}

This paper is the second in a series (cf. [13]) which concerns finite element approximations of the following fourth-order problem:

$$
\begin{aligned}
\delta \square^{2} u-\Delta u=f & \text { in } \Omega, \\
u=\partial_{\bar{n}} u=0 & \text { on } \partial \Omega,
\end{aligned}
$$

where

$$
\begin{array}{ll}
0<\delta \ll 1, & \square u:=\partial_{x x} u-\partial_{y y} u, \\
\square^{2} u:=\square(\square u), & \bar{n}:=\left(n_{1},-n_{2}\right) .
\end{array}
$$

Here, $\Omega \subset \mathbf{R}^{2}$ is an open and bounded domain, $n:=\left(n_{1}, n_{2}\right)$ is the outward unit normal to $\partial \Omega$, and $\partial_{\bar{n}} u:=\nabla u \cdot \bar{n}$. As the d'Alembertian $\square$ is the two-dimensional wave operator, we shall henceforth call $\square^{2}$ the bi-wave operator throughout the paper.

Equation (1.1) is obtained from the Ginzburg-Landau-type $d$-wave model considered in [8] (also see [24, 18]) in the absence of an applied magnetic field by neglecting the zeroth order nonlinear terms but retaining the leading terms. In the equation, $u$

Received by the editor April 10, 2009 and, in revised form, March 24, 2010.

2010 Mathematics Subject Classification. Primary 65N30, 65N12, 65N15.

Key words and phrases. Bi-wave equation, $d$-wave superconductor, Morley-type nonconforming element, discontinuous Galerkin method, error estimate.

(C) 2010 American Mathematical Society
Reverts to public domain 28 years from publication 
denotes the $d$-wave order parameter. We note that the original order parameter in the Ginzburg-Landau-type model [24, 8] is a complex-valued scalar function whose magnitude represents the density of superconducting charge carriers. However, to reduce the technicalities and to present our ideas, we assume that $u$ is a real-valued scalar function in this paper and remark that the finite element methods developed in this paper can be easily extended to the complex case. We also note that the parameter $\delta$ appears in the full model as $\delta=-\frac{1}{\beta}$, where $\beta$ is proportional to the ratio $\frac{\ln \left(T_{s 0} / T\right)}{\ln \left(T_{d 0} / T\right)}$, with $T_{s 0}$ and $T_{d 0}$ being the critical temperatures of the $s$-wave and $d$-wave components. Clearly, $\beta<0$ (or $\delta>0$ ) when $T_{s 0}<T<T_{d 0}$ and $\beta \rightarrow-\infty$ (or $\delta \rightarrow 0$ ) as $T \rightarrow T_{d 0}$. Hence, $\delta$ is expected to be small for $d$-wave like superconductors. We refer the reader to [8, 18, 24, 11] and the references therein for a detailed exposition on modeling and analysis of $d$-wave superconductors.

In [13, the authors developed two conforming finite element methods for (1.1)(1.2), and showed that unless special meshes are used, conforming finite elements are necessarily $C^{1}$ elements. Consequently, conforming plate elements such as Argyris, Bell, Hsieh-Clough-Tocher, Bogner-Fox-Schmit elements (cf. [7]) must be used in the case of general meshes. Since these finite elements require either the use of fifth or higher order polynomials or the use of exotic and complicated elements, it would be expensive and less efficient to solve the bi-wave equation (1.1) in such a fashion. This is the main motivation to construct low-order nonconforming finite element and discontinuous Galerkin methods.

The primary goal of this paper is to develop nonconforming and discontinuous Galerkin methods for problem (1.1)-(1.2). One may readily verify that

$$
\square^{2} u=\frac{\partial^{4} u}{\partial x^{4}}-2 \frac{\partial^{4} u}{\partial x^{2} \partial y^{2}}+\frac{\partial^{4} u}{\partial y^{4}} .
$$

As a comparison, the biharmonic operator $\Delta^{2}$ is defined as

$$
\Delta^{2} u:=\frac{\partial^{4} u}{\partial x^{4}}+2 \frac{\partial^{4} u}{\partial x^{2} \partial y^{2}}+\frac{\partial^{4} u}{\partial y^{4}} .
$$

Although the difference between the bi-wave and biharmonic operator is subtle, they are fundamentally different operators, as $\square^{2}$ is a hyperbolic operator, while $\Delta^{2}$ is an elliptic operator. However, it does seem possible to use various finite element methods for the biharmonic problem as a guide to construct numerical methods for the bi-wave problem. This is exactly the approach we take. The first half of this paper is devoted to the study of a nonconforming finite element method for equation (1.1), where we construct a variant of the Morley element (which is used for the biharmonic equation) that is naturally associated with the bi-wave problem. We then define the finite element method based on this new element, which unlike the biharmonic equation, requires additional jump terms to guarantee convergence. The second half of the paper is devoted to the construction and analysis of a family of symmetric interior penalty discontinuous Galerkin methods for problem (1.1) - (1.2), which is closely related to the method in 3] (also see [12]) for the biharmonic problem. The discontinuous Galerkin methods we develop are natural extensions of the Morley-type nonconforming method, as penalty terms must be used in the method for convergence. As expected, the proposed discontinuous Galerkin methods are very flexible, in particular, they do not impose any constraint on the mesh for stability and convergence. Furthermore, they allow the use of low order polynomial (quadratic or higher to be specific) trial and test functions. 
The remainder of the paper is organized as follows. In Section2, we state preliminary results concerning the well-posedness of the bi-wave problem and regularity estimates of the weak solution established in [13. Section 3 is devoted to the construction of the new Morley-type finite element and the finite element method for (1.1) using this element. We first construct the new finite element and state certain properties of the element. We then define the finite element method and prove nearly optimal order (off by a factor $|\ln h|$ ) error estimates in the energy norm and in the $H^{1}$-norm. In Section 4, we state the discontinuous Galerkin method and derive optimal order error estimates in the energy norm when the solution to (1.1) - (1.2) belongs to $H^{4}(\Omega)$. We then prove optimal order error estimates when the solution is only $H^{3}(\Omega)$ and quadratic polynomials are used. Finally, in Section 5. we present some numerical experiments to gauge the efficiency of the proposed finite element methods and to validate our theoretical error bounds.

\section{Preliminaries}

Standard function space notation is adopted in this paper. We refer the reader to [6, 7 for their exact definitions. In addition, $(\cdot, \cdot)$ and $\langle\cdot, \cdot\rangle_{\partial \Omega}$ are used to denote the $L^{2}$-inner products on $\Omega$ and $\partial \Omega$, respectively, and $C$ will denote a generic $\delta$ and $h$-independent constant that may take different values at different appearances. We also introduce the following additional space notation and norm associated with problem (1.1)-(1.2):

$$
\begin{array}{ll}
V:=\left\{v \in H^{1}(\Omega) ; \square v \in L^{2}(\Omega)\right\}, & V_{0}:=\left\{v \in V \cap H_{0}^{1}(\Omega) ;\left.\partial_{\bar{n}} v\right|_{\partial \Omega}=0\right\}, \\
(v, w)_{V}:=\delta(\square v, \square w)+(\nabla v, \nabla w), & \|v\|_{V}:=\sqrt{(v, v)_{V}}, \\
\overline{|\nabla v|}:=\nabla v \cdot \bar{\nabla} v, & \bar{\nabla} v:=\left(\partial_{x} v,-\partial_{y} v\right) .
\end{array}
$$

It is clear that $V$ endowed with the inner product $(\cdot, \cdot)$ is a Hilbert space, but we note that this is not the case if the harmonic term $\Delta u$ is removed in (1.1), since kernels of the bi-wave operator $\square^{2}$ and the wave operator $\square$ may contain nonzero functions satisfying the homogeneous Dirichlet boundary condition (see [4).

The variational formulation of (1.1) (1.2) is defined as seeking $u \in V_{0}$ such that

$$
A^{\delta}(u, v)=\langle f, v\rangle \quad \forall v \in V_{0},
$$

where

$$
A^{\delta}(u, v):=(u, v)_{V}
$$

and $\langle\cdot, \cdot\rangle$ represents the pairing between $V$ and its dual $V^{*}$.

The following theorem concerns the well-posedness of the variational formulation (2.1). A proof of the theorem can be found in [13.

Theorem 2.1. There exists a unique solution to (2.1). Furthermore,

$$
\|u\|_{V} \leq\|f\|_{V^{*}}
$$

where

$$
\|f\|_{V^{*}}=\sup _{0 \neq v \in V} \frac{\langle f, v\rangle}{\|v\|_{V}} .
$$

Moreover, if the boundary $\partial \Omega$ of the domain $\Omega$ is sufficiently smooth, there exist constants $M_{m}>0(m \geq 0)$ such that the weak solution $u$ of (2.1) satisfies

$$
\sqrt{\delta}\left|\square^{2} u\right|_{H^{m}}+\sqrt{\delta}|\nabla \square u|_{H^{m}}+|\Delta u|_{H^{m}} \leq M_{m}|f|_{H^{m}} \quad \text { if } f \in H^{m}(\Omega) .
$$


We end this section by stating some trace and inverse inequalities [3, 7, 6] which we will use later in the paper.

Lemma 2.1. Let $D \subset \mathbf{R}^{d}(d \geq 2)$ be a regular and star-like domain, and let $\rho=\operatorname{diam}(D)$. Then there exists a $D$-independent constant $C>0$ such that

(i) $\|v\|_{L^{2}(\partial D)} \leq C\left[\|v\|_{L^{2}(D)}^{\frac{1}{2}}\|\nabla v\|_{L^{2}(D)}^{\frac{1}{2}}+\rho^{-\frac{1}{2}}\|v\|_{L^{2}(D)}\right] \quad \forall v \in H^{1}(D)$,

(ii) $\quad\|v\|_{L^{2}(\partial D)} \leq C\left[\rho^{\frac{(p-2) d}{4 p}}\|v\|_{L^{p}(D)}^{\frac{1}{2}}\|\nabla v\|_{L^{2}(D)}^{\frac{1}{2}}+\rho^{\frac{(q-2) d}{2 q}-\frac{1}{2}}\|v\|_{L^{q}(D)}\right]$

$$
\forall v \in H^{1}(D), 2 \leq p, q \leq \frac{2 d}{d-2} \text { if } d \geq 3 ; 2 \leq p, q<\infty \text { if } d=2,
$$

(iii) $\|v\|_{L^{2}(\partial D)} \leq C \rho^{-\frac{1}{2}}\|v\|_{L^{2}(D)} \quad \forall v \in \mathbb{P}_{r}(D)$,

where $\mathbb{P}_{r}(D)$ denotes the space of polynomials of total degree not exceeding $r$ in $D$.

Remark 2.1. We note that inequalities (i) and (iii) are very often seen and used in the literature. However, to the best of the authors' knowledge, inequality (ii) is rarely seen in the literature, although it can be proved easily by the standard scaling argument [7, 6].

\section{A Morley-type nonconforming Finite Element method}

3.1. Construction of the nonconforming finite element. In this section, we define a new nonconforming finite element for problem (1.1)-(1.2). This new element has similar properties to that of the Morley element which is a nonconforming finite element for plate bending problems [22, 17, 20. First, we introduce the following notation, which will be useful in both this section and the next section.

Let $\mathcal{T}_{h}$ be a quasi-uniform triangulation of $\Omega$ with mesh size $h \in(0,1)$. For a given $T \in \mathcal{T}_{h}$, let $\left(\lambda_{1}^{T}, \lambda_{2}^{T}, \lambda_{3}^{T}\right)$ denote the associated barycentric coordinates, and $a_{i}(1 \leq i \leq 3)$ denote the vertices of $T$. Let $e_{i}(1 \leq i \leq 3)$ denote the edge of $T$ of which $a_{i}$ is not a vertex. Next, we define the following sets of edges:

$$
\mathcal{E}_{h}^{I}:=\{e ; e \cap \partial \Omega=\emptyset\}, \quad \mathcal{E}_{h}^{B}:=\{e ; e \cap \partial \Omega \neq \emptyset\}, \quad \mathcal{E}_{h}:=\mathcal{E}_{h}^{I} \cup \mathcal{E}_{h}^{B} .
$$

For any $e \in \mathcal{E}_{h}^{I}$, there exists $T_{1}, T_{2} \in \mathcal{T}_{h}$ such that $e=T_{1} \cap T_{2}$. For $v \in$ $H^{1}\left(T_{1}\right) \cap H^{1}\left(T_{2}\right)$, define the jumps and averages of $v$ on $e$ by

$$
\left.[v]\right|_{e}=\left.v^{T_{1}}\right|_{e}-\left.v^{T_{2}}\right|_{e},\left.\quad\{v\}\right|_{e}=\frac{1}{2}\left(\left.v^{T_{1}}\right|_{e}+\left.v^{T_{2}}\right|_{e}\right),
$$

where $v^{T_{i}}=\left.v\right|_{T_{i}}$. For $v \in H^{2}\left(T_{1}\right) \cap H^{2}\left(T_{2}\right), \alpha \in \mathbf{R}^{2}$, we define the jumps and averages of $\partial_{\alpha} v:=\nabla v \cdot \alpha$ on $e$ by

$$
\left.\left[\partial_{\alpha} v\right]\right|_{e}=\left.\partial_{\alpha} v^{T_{1}}\right|_{e}-\left.\partial_{\alpha} v^{T_{2}}\right|_{e},\left.\quad\left\{\partial_{\alpha} v\right\}\right|_{e}=\frac{1}{2}\left(\left.\partial_{\alpha} v^{T_{1}}\right|_{e}+\left.\partial_{\alpha} v^{T_{2}}\right|_{e}\right),
$$

and for $v \in H^{3}\left(T_{1}\right) \cap H^{3}\left(T_{2}\right), \alpha, \beta \in \mathbf{R}^{2}$, we define the jumps and averages of $\partial_{\alpha \beta} v:=D^{2} v \alpha \cdot \beta$ (where throughout the paper, $D^{2} v$ denotes the Hessian of $v$ ) on $e$ as follows:

$$
\left.\left[\partial_{\alpha \beta} v\right]\right|_{e}=\left.\partial_{\alpha \beta} v^{T_{1}}\right|_{e}-\left.\partial_{\alpha \beta} v^{T_{2}}\right|_{e},\left.\quad\left\{\partial_{\alpha \beta} v\right\}\right|_{e}=\frac{1}{2}\left(\left.\partial_{\alpha \beta} v^{T_{1}}\right|_{e}+\left.\partial_{\alpha \beta} v^{T_{2}}\right|_{e}\right) .
$$

For any $e \in \mathcal{E}_{h}^{B}$, there is a triangle $T_{1} \in \mathcal{T}_{h}$ such that $e=\partial T_{1} \cap \partial \Omega$. We then define the jumps and averages of $v, \partial_{\alpha} v$, and $\partial_{\alpha \beta} v$ (assuming such quantities are 

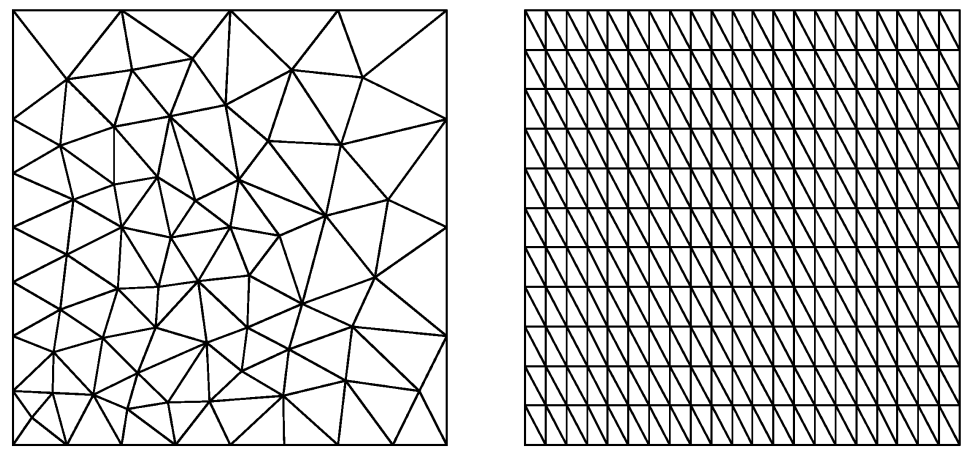

FiguRE 1. Example of a nonuniform mesh (left) and a uniform mesh (right) of the domain $\Omega=(0,1)^{2}$ such that every triangle has no type I edge.

defined) as follows:

$$
\begin{array}{ll}
{\left.[v]\right|_{e}=\left.v^{T_{1}}\right|_{e},} & \left.\{v\}\right|_{e}=\left.v^{T_{1}}\right|_{e}, \\
{\left.\left[\partial_{\alpha} v\right]\right|_{e}=\left.v^{T_{1}}\right|_{e},} & \left.\left\{\partial_{\alpha} v\right\}\right|_{e}=\left.v^{T_{1}}\right|_{e}, \\
{\left.\left[\partial_{\alpha \beta} v\right]\right|_{e}=\left.\partial_{\alpha \beta} v^{T_{1}}\right|_{e},} & \left.\left\{\partial_{\alpha \beta} v\right\}\right|_{e}=\left.\partial_{\alpha \beta} v^{T_{1}}\right|_{e} .
\end{array}
$$

In the rest of this section, we shall often encounter the following shape characterization of the meshes.

Definition 3.1. For $e \in \mathcal{E}_{h}$, let $n$ and $\tau$ denote the outward unit normal and unit tangent vector of $e$, respectively. We say that $e$ is a type $I$ edge if

$$
\bar{n}=\tau \quad \text { or } \quad \bar{n}=-\tau \text {. }
$$

Otherwise, $e$ is called a type II edge if condition (3.1) does not hold.

Remark 3.1. (a) We note that if $e$ is a type I edge, then $\bar{n}=\left(n_{1},-n_{2}\right)= \pm \tau=$ $\pm\left(\tau_{1}, \tau_{2}\right)= \pm\left(n_{2},-n_{1}\right)$. Therefore, we conclude

$$
\tau=\frac{\sqrt{2}}{2}( \pm 1, \pm 1)
$$

That is, the edge $e$ makes an angle of $\frac{\pi}{4}$ in the plane with respect to the $x$-axis. Examples of meshes such that every triangle in the partition has no type I edge are shown in Figure 1 .

(b) For $T \in \mathcal{T}_{h}, e_{i} \subset \partial T$, let $n^{(i)}, \tau^{(i)}$ denote the outward (from $T$ ) unit normal and unit tangent vector of $e_{i}$, respectively. Then using the identity $n^{(i)}=-\frac{\nabla \lambda_{i}^{T}}{\left\|\nabla \lambda_{i}^{T}\right\|}$, $e_{i}$ is a type I edge if and only if

$$
\overline{\left|\nabla \lambda_{i}^{T}\right|}=0 .
$$

We now define a new finite element $S_{2}^{h}=\left(T, P_{T}, \Sigma_{T}\right)$ as follows:

(i) $T$ is a triangle with no type I edge,

(ii) $P_{T}=\mathbb{P}_{2}(T)$, the space of quadratic polynomials,

(iii) $\Sigma_{T}= \begin{cases}v\left(a_{i}\right), & 1 \leq i \leq 3, \\ \partial_{\bar{n}} v\left(a_{i j}\right), & 1 \leq i<j \leq 3,\end{cases}$ 


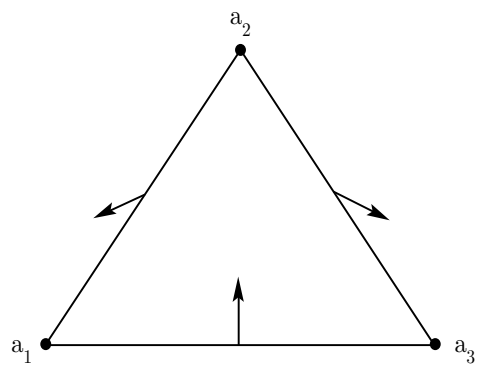

Figure 2. Element $S_{2}^{h}$. Solid dots indicate function evaluation and arrows indicate evaluation of derivatives in the direction $\bar{n}$

where $a_{i j}=\frac{1}{2}\left(a_{i}+a_{j}\right)$.

The reason to restrict the element such that $T$ has no type I edge is explained in the following lemma.

Lemma 3.1. Suppose $T \in \mathcal{T}_{h}$ has a type I edge in the set $\mathcal{E}_{h}$. Then $\Sigma_{T}$ is not linearly independent, and hence, $S_{2}^{h}$ is not unisolvent on $T$.

Proof. Let $e_{3}$ denote the type I edge of $T$. For $v \in P_{T}$, write

$$
\left.v\right|_{T}=c_{1}\left(\lambda_{1}^{T}\right)^{2}+c_{2}\left(\lambda_{2}^{T}\right)^{2}+c_{3} \lambda_{1}^{T} \lambda_{2}^{T}+c_{4} \lambda_{1}^{T}+c_{5} \lambda_{2}^{T}+c_{6} .
$$

We then have

$$
\begin{aligned}
v\left(a_{3}\right) & =c_{6} \\
v\left(a_{2}\right) & =c_{2}+c_{5}+v\left(a_{3}\right) \\
v\left(a_{1}\right) & =c_{1}+c_{4}+v\left(a_{3}\right), \\
\partial_{\bar{n}} v\left(a_{12}\right) & =-\left(\left(c_{1}+c_{4}\right) \nabla \lambda_{1}^{T}+\left(c_{2}+c_{5}\right) \nabla \lambda_{2}^{T}+\frac{c_{3}}{2}\left(\nabla \lambda_{1}^{T}+\nabla \lambda_{2}^{T}\right)\right) \cdot \frac{\bar{\nabla} \lambda_{3}^{T}}{\left\|\nabla \lambda_{3}^{T}\right\|} .
\end{aligned}
$$

Noting

$$
\left(\nabla \lambda_{1}^{T}+\nabla \lambda_{2}^{T}\right) \cdot \bar{\nabla} \lambda_{3}^{T}=-\overline{\left|\nabla \lambda_{3}^{T}\right|}=0
$$

we obtain

$$
\partial_{\bar{n}} v\left(a_{12}\right)=\left(\left(v\left(a_{3}\right)-v\left(a_{1}\right)\right) \nabla \lambda_{1}^{T}+\left(v\left(a_{3}\right)-v\left(a_{2}\right)\right) \nabla \lambda_{2}^{T}\right) \cdot \frac{\bar{\nabla} \lambda_{3}^{T}}{\left\|\nabla \lambda_{3}^{T}\right\|} .
$$

Therefore, the degree of freedom $\partial_{\bar{n}} v\left(a_{12}\right)$ is redundant in $\Sigma_{T}$.

Remark 3.2. In contrast, the two finite elements constructed in 13 for problem (1.1)-(1.2) require that every triangle $T \in \mathcal{T}_{h}$ have exactly two type I edges, which can be considered the opposite mesh restriction that is required to use the Morleytype element $S_{2}^{h}$. 
Remark 3.3. The basis functions associated with the element $S_{2}^{h}=\left(T, P_{T}, \Sigma_{T}\right)$ are given by

$$
\begin{array}{rlrl}
\varphi_{j}^{T}:= & \frac{\left\|\nabla \lambda_{j}^{T}\right\|}{\overline{\left|\nabla \lambda_{j}^{T}\right|}} \lambda_{j}^{T}\left(\lambda_{j}^{T}-1\right), & 1 \leq j \leq 3, \\
\varphi_{i j}^{T}:=1-\left(\lambda_{i}^{T}+\lambda_{j}^{T}\right)+2 \lambda_{i}^{T} \lambda_{j}^{T} & \\
& \quad-\nabla \lambda_{i}^{T} \cdot \bar{\nabla} \lambda_{j}^{T}\left(\frac{\varphi_{i}^{T}}{\left\|\nabla \lambda_{i}^{T}\right\|}+\frac{\varphi_{j}^{T}}{\left\|\nabla \lambda_{j}^{T}\right\|}\right), & 1 \leq i<j \leq 3 .
\end{array}
$$

We note that if we replace $\bar{\nabla}(\cdot)$ with $\nabla(\cdot)$ and $\overline{|\nabla(\cdot)|}$ with $\|\nabla(\cdot)\|^{2}$, we obtain the standard basis functions for the Morley element [17, 22].

3.2. Properties of the new finite element. Let $V^{h}$ be the corresponding finite element space to the element $S_{2}^{h}$, and let $V_{0}^{h}$ consist of the functions in $V^{h}$ whose degrees of freedom vanish on $\partial \Omega$, that is,

$V^{h}=\left\{\left.v\right|_{T} \in \mathbb{P}_{2}(T) ; v\right.$ is continuous at each degree of freedom in $\left.\Sigma_{T}, \forall T \in \mathcal{T}_{h}\right\}$, $V_{0}^{h}=\left\{v \in V^{h} ; v\right.$ vanishes at all degrees of freedom on $\left.\partial \Omega\right\}$.

Next, define the following broken Sobolev norms and semi-norms

$$
\|v\|_{m, h}^{2}:=\sum_{T \in \mathcal{T}_{h}}\|v\|_{H^{m}(T)}^{2}, \quad|v|_{m, h}^{2}:=\sum_{T \in \mathcal{T}_{h}}|v|_{H^{m}(T)}^{2},
$$

and it is understood that $\|v\|_{0, h}=\|v\|_{L^{2}}$.

Let $\Pi_{T} v$ denote the standard interpolation of $v$ associated with the finite element $S_{2}^{h}$, that is,

$$
\Pi_{T} v=\sum_{1 \leq i<j \leq 3, k \neq i, j} \varphi_{i j}^{T} v\left(a_{k}\right)+\sum_{j=1}^{3} \varphi_{j}^{T} \partial_{\bar{n}} v\left(a_{j}\right) .
$$

We also define $\Pi_{h} v \in V^{h}$ such that $\left.\Pi_{h} v\right|_{T}=\Pi_{T}\left(\left.v\right|_{T}\right), \forall T \in \mathcal{T}_{h}$. We note that $\Pi_{T} v=v \forall v \in \mathbb{P}_{2}(T)$, and therefore, using standard interpolation theory [7] we have for $0 \leq m \leq 3$,

$$
\left|v-\Pi_{T} v\right|_{H^{m}(T)} \leq C h^{3-m}|v|_{H^{3}(T)} \quad \forall v \in H^{3}(T), T \in \mathcal{T}_{h} .
$$

Next, we show that the finite element space $V^{h}$ inherits a form of "weak continuity" which will play a crucial role in our analysis.

Lemma 3.2. For all $\alpha \in \mathbf{R}^{2}$,

$$
\begin{array}{cc}
\int_{e}\left[\partial_{\alpha} v\right] d s=0 & \forall v \in V^{h}, \quad e \in \mathcal{E}_{h}^{I}, \\
\int_{e} \partial_{\alpha} v d s=0 & \forall v \in V_{0}^{h}, \quad e \in \mathcal{E}_{h}^{B} .
\end{array}
$$

Proof. Given $e \in \mathcal{E}_{h}$, let $a_{1}, a_{2}$ denote the endpoints of $e, a_{12}$ the midpoint of $e$, and $n, \tau$ the normal and tangential direction of $e$. By hypothesis $\bar{n} \neq \pm \tau$. Thus, 
we can write for any constant vector $\alpha \in \mathbf{R}^{2}$,

$$
\begin{gathered}
\int_{e} \partial_{\alpha} v d s=\frac{1}{1-(\tau \cdot \bar{n})^{2}} \int_{e}\left(\alpha \cdot(\tau-\bar{n}(\tau \cdot \bar{n})) \partial_{\tau} v+\alpha \cdot(\bar{n}-\tau(\tau \cdot \bar{n})) \partial_{\bar{n}} v\right) d s \\
=\frac{1}{1-(\tau \cdot \bar{n})^{2}}\left(\alpha \cdot(\tau-\bar{n}(\tau \cdot \bar{n}))\left(v\left(a_{2}\right)-v\left(a_{1}\right)\right)\right. \\
\left.+\alpha \cdot(\bar{n}-\tau(\tau \cdot \bar{n})) \partial_{\bar{n}} v\left(a_{12}\right)\right) .
\end{gathered}
$$

From this identity, the desired result follows.

From the above "weak continuity" result, we get the following lemmas (see [22]).

Lemma 3.3. Let $e \in \mathcal{E}_{h}$. Then if $e=\partial T_{1} \cap \partial T_{2}$ for some $T_{1}, T_{2} \in \mathcal{T}_{h}$,

$$
\|[v]\|_{L^{2}(e)}+h\|[\nabla v]\|_{L^{2}(e)} \leq C h^{\frac{3}{2}}\left(|v|_{H^{2}\left(T_{1}\right)}+|v|_{H^{2}\left(T_{2}\right)}\right) \quad \forall v \in V^{h},
$$

and if $e=\partial T_{1} \cap \partial \Omega$ for some $T_{1} \in \mathcal{T}_{h}$,

$$
\|[v]\|_{L^{2}(e)}+h\|[\nabla v]\|_{L^{2}(e)} \leq C h^{\frac{3}{2}}|v|_{H^{2}\left(T_{1}\right)} \quad \forall v \in V_{0}^{h} .
$$

Lemma 3.4. For every $v \in V_{0}^{h}$, there exist functions $v_{k} \in H_{0}^{1}(\Omega) k=0,1,2$ with $\left.v_{k}\right|_{T} \in \mathbb{P}_{1}(T)$ such that

$$
\begin{array}{rlrl}
\left|v-v_{0}\right|_{m, h} & \leq C h^{2-m}|v|_{2, h} & & 0 \leq m \leq 2, \\
\left|\partial_{x_{k}} v-v_{k}\right|_{m, h} \leq C h^{1-m}|v|_{2, h} & & 0 \leq m \leq 1, \quad k=1,2 .
\end{array}
$$

Corollary 3.1. $|\cdot|_{m, h}$ and $\|\cdot\|_{m, h}$ are equivalent on $V_{0}^{h}$ for $m=0,1,2$.

Proof. Using the inverse inequality and Poincaré's inequality, we have

$$
\begin{aligned}
\|v\|_{L^{2}} & \leq\left\|v_{0}\right\|_{L^{2}}+\left\|v-v_{0}\right\|_{L^{2}} \leq\left|v_{0}\right|_{H^{1}}+C h^{2}|v|_{2, h} \\
& \leq C|v|_{1, h}+C h|v|_{1, h} \leq C|v|_{1, h} \quad \forall v \in V_{0}^{h} .
\end{aligned}
$$

Similarly, for $k=1,2$,

$$
\left\|\partial_{x_{k}} v\right\|_{L^{2}} \leq\left\|v_{k}\right\|_{L^{2}}+\left\|\partial_{x_{k}} v-v_{k}\right\|_{L^{2}} \leq\left|v_{k}\right|_{H^{1}}+C h|v|_{2, h} \leq C|v|_{2, h} .
$$

From these two identities, the desired results follow.

3.3. Formulation and convergence analysis of the Morley-type nonconforming method. Based on (2.1), we define our nonconforming finite element method as seeking $u_{h} \in V_{0}^{h}$ such that

$$
A_{h}^{\delta}\left(u_{h}, v\right)=(f, v) \quad \forall v \in V_{0}^{h},
$$

where

$$
\begin{aligned}
A_{h}^{\delta}(v, w)= & \sum_{T \in \mathcal{T}_{h}}\left(\delta(\square v, \square w)_{T}+(\nabla v, \nabla w)_{T}\right) \\
& +\sum_{e \in \mathcal{E}_{h}} \gamma h|\ln h|\left(\left\langle\left[\partial_{\bar{\tau} \bar{\tau}} v\right],\left[\partial_{\bar{\tau} \bar{\tau}} w\right]\right\rangle_{e}+\left\langle\left[\partial_{\bar{\tau} \bar{n}} v\right],\left[\partial_{\bar{\tau} \bar{n}} w\right]\right\rangle_{e}\right) .
\end{aligned}
$$

Recall that $\tau=\left(\tau_{1}, \tau_{2}\right)$ and $n=\left(n_{1}, n_{2}\right)$ denote the unit tangent and outward unit normal to $e, \bar{\tau}:=\left(\tau_{1},-\tau_{2}\right)=\left(n_{2}, n_{1}\right)$, and $h_{e}=|e|$, the length of $e$. Also, $(\cdot, \cdot)_{T}$ 
and $\langle\cdot, \cdot\rangle_{e}$ denote the $L^{2}$ inner product on $T$ and $e$, respectively. We note that $A_{h}^{\delta}(\cdot, \cdot)$ induces the following energy norm on $V_{0}^{h}$ :

$$
\begin{aligned}
\|v\|_{M}^{2}:=\sum_{T \in \mathcal{T}_{h}}\left(\delta\|\square v\|_{L^{2}(T)}^{2}\right. & \left.+\|\nabla v\|_{L^{2}(T)}^{2}\right) \\
& +\sum_{e \in \mathcal{E}_{h}} \gamma h|\ln h|\left(\left\|\left[\partial_{\bar{\tau} \bar{\tau}} v\right]\right\|_{L^{2}(e)}^{2}+\left\|\left[\partial_{\bar{\tau} \bar{n}} v\right]\right\|_{L^{2}(e)}^{2}\right) .
\end{aligned}
$$

Remark 3.4. In the finite element method (3.2), the jump terms are the so-called penalty terms. The reason to include penalty terms into the finite element method is to ensure that $\|\cdot\|_{M}$ is equivalent (independent of $h$ ) to the broken Sobolev norm $\|\cdot\|_{2, h}$ on the space $V_{0}^{h}$ (see Lemma 3.6). We note that the basis functions $\varphi_{i j}^{T}$ satisfy (cf. Remark 3.3)

$$
\square \varphi_{i j}^{T}=0 \quad \forall T \in \mathcal{T}_{h}, \quad 1 \leq i<j \leq 3 .
$$

Therefore, equivalence of $\|\cdot\|_{M}$ and $\|\cdot\|_{2, h}$ cannot be obtained in the absence of penalty terms.

For clarity of the presentation, we also introduce the following additional notation:

$$
\begin{aligned}
S_{T}(v, w) & :=\left\langle\partial_{\bar{\tau} \bar{\tau}} v, \partial_{n} w\right\rangle_{\partial T}-\left\langle\partial_{\overline{\bar{\tau}}} v, \partial_{\tau} w\right\rangle_{\partial T}, \\
Q_{T}(v, w) & :=2\left(\partial_{x y} v, \partial_{x y} w\right)_{T}+\left(\partial_{x x} v, \partial_{y y} w\right)_{T}+\left(\partial_{y y} v, \partial_{x x} w\right)_{T}, \\
S(v, w) & :=\sum_{T \in \mathcal{T}_{h}} S_{T}(v, w) \\
Q(v, w) & :=\sum_{T \in \mathcal{T}_{h}} Q_{T}(v, w) .
\end{aligned}
$$

Before proving the main results of this section, we first establish two technical lemmas.

Lemma 3.5. For any $T \in \mathcal{T}_{h}$ and any two smooth functions $v$ and $w$ on $T$, the following identity holds:

$$
\begin{aligned}
S_{T}(v, w)=\left\langle\partial_{x y} v, \partial_{x} w n_{2}\right\rangle_{\partial T} & +\left\langle\partial_{x y} v, \partial_{y} w n_{1}\right\rangle_{\partial T} \\
& +\left\langle\partial_{y y} v, \partial_{x} w n_{1}\right\rangle_{\partial T}+\left\langle\partial_{x x} v, \partial_{y} w n_{2}\right\rangle_{\partial T} .
\end{aligned}
$$

Proof. By definition, we have

$$
\begin{aligned}
S_{T}(v, w)= & \left\langle\partial_{\bar{\tau} \bar{\tau}} v, \partial_{n} w\right\rangle_{\partial T}-\left\langle\partial_{\bar{\tau} \bar{n}} v, \partial_{\tau} w\right\rangle_{\partial T} \\
= & \left\langle\partial_{x x} v \bar{\tau}_{1}^{2}+2 \partial_{x y} v \bar{\tau}_{1} \bar{\tau}_{2}+\partial_{y y} v \bar{\tau}_{2}^{2}, \partial_{x} w n_{1}+\partial_{y} w n_{2}\right\rangle_{\partial T}-\left\langle\partial_{x x} v \bar{\tau}_{1} \bar{n}_{1}\right. \\
& \left.\quad+\partial_{x y} v \bar{n}_{1} \bar{\tau}_{2}+\partial_{x y} v \bar{\tau}_{1} \bar{n}_{2}+\partial_{y y} v \bar{\tau}_{2} \bar{n}_{2}, \partial_{x} w \tau_{1}+\partial_{y} w \tau_{2}\right\rangle_{\partial T} .
\end{aligned}
$$

Since $\left(\bar{n}_{1}, \bar{n}_{2}\right)=\left(n_{1},-n_{2}\right)$ and $\left(\bar{\tau}_{1}, \bar{\tau}_{2}\right)=\left(n_{2}, n_{1}\right)$,

$$
\begin{aligned}
S_{T}(v, w)= & \left\langle\partial_{x x} v n_{2}^{2}+2 \partial_{x y} v n_{1} n_{2}+\partial_{y y} v n_{1}^{2}, \partial_{x} w n_{1}+\partial_{y} w n_{2}\right\rangle_{\partial T} \\
& -\left\langle\partial_{x x} v n_{1} n_{2}+\partial_{x y} v n_{1}^{2}-\partial_{x y} v n_{2}^{2}-\partial_{y y} v n_{2} n_{1}, \partial_{x} w n_{2}-\partial_{y} w n_{1}\right\rangle_{\partial T}
\end{aligned}
$$


Finally, expanding the last expression and grouping similar terms, we conclude

$$
\begin{aligned}
S_{T}(v, w)=\langle & \left.\partial_{x} w, \partial_{x x} v n_{2}^{2} n_{1}+2 \partial_{x y} v n_{1}^{2} n_{2}+\partial_{y y} v n_{1}^{3}-\partial_{x x} v n_{1} n_{2}^{2}\right\rangle_{\partial T} \\
& +\left\langle\partial_{x} w, \partial_{x y} v n_{2}^{3}-\partial_{x y} v n_{1}^{2} n_{2}+\partial_{y y} v n_{1} n_{2}^{2}\right\rangle_{\partial T} \\
& +\left\langle\partial_{y} w, \partial_{x x} v n_{2}^{3}+2 \partial_{x y} v n_{1} n_{2}^{2}+\partial_{y y} v n_{1}^{2} n_{2}+\partial_{x x} v n_{1}^{2} n_{2}\right\rangle_{\partial T} \\
& +\left\langle\partial_{y} w, \partial_{x y} v n_{1}^{3}-\partial_{x y} v n_{2}^{2} n_{1}-\partial_{y y} v n_{2} n_{1}^{2}\right\rangle_{\partial T} \\
=\langle & \left.\partial_{x} w, \partial_{x y} v n_{2}\left(n_{1}^{2}+n_{2}^{2}\right)+\partial_{y y} v n_{1}\left(n_{1}^{2}+n_{2}^{2}\right)\right\rangle_{\partial T} \\
& +\left\langle\partial_{y} w, \partial_{x x} v n_{2}\left(n_{1}^{2}+n_{2}^{2}\right)+\partial_{x y} v n_{1}\left(n_{1}^{2}+n_{2}^{2}\right)\right\rangle_{\partial T} \\
=\langle & \left.\partial_{x y} v, \partial_{x} w n_{2}\right\rangle_{\partial T}+\left\langle\partial_{x y} v, \partial_{y} w n_{1}\right\rangle_{\partial T} \\
& +\left\langle\partial_{x x} v, \partial_{y} w n_{2}\right\rangle_{\partial T}+\left\langle\partial_{y y} v, \partial_{x} w n_{1}\right\rangle_{\partial T} .
\end{aligned}
$$

Corollary 3.2. For any $T \in \mathcal{T}_{h}, v, w \in \mathbb{P}_{2}(T)$, the following identity holds:

$$
Q_{T}(v, w)=S_{T}(v, w)
$$

Proof. Since $v,\left.w\right|_{T} \in \mathbb{P}_{2}(T)$, integrating by parts and applying Lemma 3.5 gives us

$$
\begin{aligned}
Q_{T}(v, w)=\langle & \left.\partial_{x y} v, \partial_{x} w n_{2}\right\rangle_{\partial T}+\left\langle\partial_{x y} v, \partial_{y} w n_{1}\right\rangle_{\partial T} \\
& +\left\langle\partial_{y y} v, \partial_{x} w n_{1}\right\rangle_{\partial T}+\left\langle\partial_{x x} v, \partial_{y} w n_{2}\right\rangle_{\partial T}=S_{T}(v, w) .
\end{aligned}
$$

Lemma 3.6. There exists a $\gamma_{0}=O(\delta)$ such that for $\gamma \geq \gamma_{0}$ the following inequality holds:

$$
\|v\|_{M}^{2} \geq \delta C\|v\|_{2, h}^{2} \quad \forall v \in V_{0}^{h}
$$

where $C$ is a positive constant independent of $\gamma$ and $h$.

Proof. We divide the proof into three steps.

Step 1. Integrating by parts and applying Corollary 3.2 yields

$$
\begin{gathered}
\|v\|_{M}^{2}=\sum_{T \in \mathcal{T}_{h}}\left(\delta\|\square v\|_{T}^{2}+\|\nabla v\|_{T}^{2}\right)+\sum_{e \in \mathcal{E}_{h}} \gamma h|\ln h|\left(\left\|\left[\partial_{\bar{\tau} \bar{\tau}} v\right]\right\|_{L^{2}(e)}^{2}+\left\|\left[\partial_{\bar{\tau} \bar{n}} v\right]\right\|_{L^{2}(e)}^{2}\right) \\
=\sum_{T \in \mathcal{T}_{h}}\left(\delta\left\|D^{2} v\right\|_{L^{2}(T)}^{2}+\|\nabla v\|_{L^{2}(T)}^{2}-\delta Q_{T}(v, v)\right) \\
\quad+\sum_{e \in \mathcal{E}_{h}} \gamma h|\ln h|\left(\left\|\left[\partial_{\bar{\tau} \bar{\tau}} v\right]\right\|_{L^{2}(e)}^{2}+\left\|\left[\partial_{\bar{\tau} \bar{n}} v\right]\right\|_{L^{2}(e)}^{2}\right) \\
=\sum_{T \in \mathcal{T}_{h}}\left(\delta\left\|D^{2} v\right\|_{L^{2}(T)}^{2}+\|\nabla v\|_{L^{2}(T)}^{2}-\delta S_{T}(v, v)\right) \\
\quad+\sum_{e \in \mathcal{E}_{h}} \gamma h|\ln h|\left(\left\|\left[\partial_{\bar{\tau} \bar{\tau}} v\right]\right\|_{L^{2}(e)}^{2}+\left\|\left[\partial_{\bar{\tau} \bar{n}} v\right]\right\|_{L^{2}(e)}^{2}\right) .
\end{gathered}
$$


Hence, by Lemma 3.2

$$
\begin{aligned}
& \|v\|_{M}^{2}=\sum_{T \in \mathcal{T}_{h}}\left(\delta\left\|D^{2} v\right\|_{T}^{2}+\|\nabla v\|_{L^{2}(T)}^{2}\right)+\sum_{e \in \mathcal{E}_{h}}\left(\gamma h | \operatorname { l n } h | \left(\left\|\left[\partial_{\bar{\tau} \bar{\tau}} v\right]\right\|_{L^{2}(e)}^{2}\right.\right. \\
& \left.+\left\|\left[\partial_{\bar{\tau} \bar{n}} v\right]\right\|_{L^{2}(e)}^{2}\right)-\delta\left(\left\langle\left[\partial_{\bar{\tau} \bar{\tau}} v\right],\left\{\partial_{n} v\right\}\right\rangle+\left\langle\left\{\partial_{\bar{\tau} \bar{\tau}} v\right\},\left[\partial_{n} v\right]\right\rangle_{e}\right. \\
& \left.\left.-\left\langle\left[\partial_{\bar{\tau} \bar{n}} v\right],\left\{\partial_{\tau} v\right\}\right\rangle_{e}-\left\langle\left\{\partial_{\bar{\tau} \bar{n}} v\right\},\left[\partial_{\tau} v\right]\right\rangle_{e}\right)\right) \\
& =\sum_{T \in \mathcal{T}_{h}}\left(\delta\left\|D^{2} v\right\|_{L^{2}(T)}^{2}+\|\nabla v\|_{L^{2}(T)}^{2}\right)+\sum_{e \in \mathcal{E}_{h}}\left(\gamma h | \operatorname { l n } h | \left(\left\|\left[\partial_{\bar{\tau} \bar{\tau}} v\right]\right\|_{L^{2}(e)}^{2}\right.\right. \\
& \left.\left.+\left\|\left[\partial_{\bar{\tau} \bar{n}} v\right]\right\|_{L^{2}(e)}^{2}\right)-\delta\left(\left\langle\left[\partial_{\bar{\tau} \bar{\tau}} v\right],\left\{\partial_{n} v\right\}\right\rangle_{e}-\left\langle\left[\partial_{\bar{\tau} \bar{n}} v\right],\left\{\partial_{\tau} v\right\}\right\rangle_{e}\right)\right) \\
& \geq \sum_{T \in \mathcal{T}_{h}}\left(\delta\left\|D^{2} v\right\|_{L^{2}(T)}^{2}+\|\nabla v\|_{L^{2}(T)}^{2}\right)+\sum_{e \in \mathcal{E}_{h}}\left(\gamma h | \operatorname { l n } h | \left(\left\|\left[\partial_{\bar{\tau} \bar{\tau}} v\right]\right\|_{L^{2}(e)}^{2}\right.\right. \\
& \left.\left.+\left\|\left[\partial_{\bar{\tau} \bar{n}} v\right]\right\|_{L^{2}(e)}^{2}\right)-\delta\left(\left|\left\langle\left[\partial_{\bar{\tau} \bar{\tau}} v\right],\left\{\partial_{n} v\right\}\right\rangle_{e}\right|+\left|\left\langle\left[\partial_{\bar{\tau} \bar{n}} v\right],\left\{\partial_{\tau} v\right\}\right\rangle_{e}\right|\right)\right) .
\end{aligned}
$$

Step 2. Next, we derive a lower bound for each of the last two terms on the righthand side of (3.5). Since $v$ is quadratic, both $\partial_{\bar{\tau} \bar{\tau}} v$ and $\partial_{\bar{\tau} \bar{n}} v$ are constants along each $e \in \mathcal{E}_{h}$. By Lemma 3.2 we have

$$
\begin{aligned}
& \left\langle\left[\partial_{\bar{\tau} \bar{\tau}} v\right],\left\{\partial_{n} v\right\}\right\rangle_{e}=\left\langle\left[\partial_{\bar{\tau} \bar{\tau}} v\right],\left\{\partial_{n} v\right\}+\frac{1}{2}\left[\partial_{n} v\right]\right\rangle_{e}=\left\langle\left[\partial_{\bar{\tau} \bar{\tau}} v\right], \partial_{n} v\right\rangle_{e}, \\
& \left\langle\left[\partial_{\bar{\tau} \bar{n}} v\right],\left\{\partial_{\tau} v\right\}\right\rangle_{e}=\left\langle\left[\partial_{\bar{\tau} \bar{n}} v\right],\left\{\partial_{\tau} v\right\}+\frac{1}{2}\left[\partial_{\tau} v\right]\right\rangle_{e}=\left\langle\left[\partial_{\bar{\tau} \bar{n}} v\right], \partial_{\tau} v\right\rangle_{e} .
\end{aligned}
$$

Let $\mathbf{e}_{1}=(1,0)^{T}$ and $\mathbf{e}_{2}=(0,1)^{T}$ denote the canonical orthogonal basis for $\mathbf{R}^{2}$. For $e \in \mathcal{E}_{h}$, let $T \in \mathcal{T}_{h}$ be the element (with larger global labeling) which has $e$ as its one edge. On noting that $\left[\partial_{\bar{\tau} \bar{\tau}} v\right]$ and $\left[\partial_{\bar{\tau} \bar{n}} v\right]$ are constants along $e$, by the quasi-uniformity of $\mathcal{T}_{h}$ we get

$$
\begin{aligned}
& \left|\left\langle\left[\partial_{\bar{\tau} \bar{\tau}} v\right],\left\{\partial_{n} v\right\}\right\rangle_{e}\right| \leq C h_{e}\left\|\left[\partial_{\bar{\tau} \bar{\tau}} v\right]\right\|_{L^{\infty}(e)}\|\nabla v\|_{L^{\infty}(T)} \\
& \quad \leq C h_{e}\left\|\left[\partial_{\bar{\tau} \bar{\tau}} v\right]\right\|_{L^{\infty}(e)}\left(\left\|\partial_{\mathbf{e}_{1}} v\right\|_{L^{\infty}(T)}+\left\|\partial_{\mathbf{e}_{2}} v\right\|_{L^{\infty}(T)}\right) \\
& \quad \leq \frac{h|\ln h|}{2 \varepsilon}\left\|\left[\partial_{\bar{\tau} \bar{\tau}} v\right]\right\|_{L^{2}(e)}^{2}+C \frac{\varepsilon}{|\ln h|}\left(\left\|\partial_{\mathbf{e}_{1}} v\right\|_{L^{\infty}(T)}^{2}+\left\|\partial_{\mathbf{e}_{2}} v\right\|_{L^{\infty}(T)}^{2}\right), \\
& \quad\left|\left\langle\left[\partial_{\bar{\tau} \bar{n}} v\right],\left\{\partial_{n} v\right\}\right\rangle_{e}\right| \leq C h_{e}\left\|\left[\partial_{\bar{\tau} \bar{n}} v\right]\right\|_{L^{\infty}(e)}\|\nabla v\|_{L^{\infty}(T)} \\
& \quad \leq C h_{e}\left\|\left[\partial_{\bar{\tau} \bar{n}} v\right]\right\|_{L^{\infty}(e)}\left(\left\|\partial_{\mathbf{e}_{1}} v\right\|_{L^{\infty}(T)}+\left\|\partial_{\mathbf{e}_{2}} v\right\|_{L^{\infty}(T)}\right) \\
& \quad \leq \frac{h|\ln h|}{2 \varepsilon}\left\|\left[\partial_{\bar{\tau} \bar{n}} v\right]\right\|_{L^{2}(e)}^{2}+C \frac{\varepsilon}{|\ln h|}\left(\left\|\partial_{\mathbf{e}_{1}} v\right\|_{L^{\infty}(T)}^{2}+\left\|\partial_{\mathbf{e}_{2}} v\right\|_{L^{\infty}(T)}^{2}\right),
\end{aligned}
$$

where $\epsilon$ is a positive number to be chosen later.

To continue, we use a discrete Sobolev-Poincaré inequality for piecewise polynomials, which was proved in 5] (also see [16] for a related inequality for piecewise 
$H^{1}$ functions), to get

$$
\begin{aligned}
\sum_{T \in \mathcal{T}_{h}}\left\|\partial_{\mathbf{e}_{i}} v\right\|_{L^{\infty}(T)}^{2} \leq C|\ln h| & {\left[\sum_{T \in \mathcal{T}_{h}}\left\|D^{2} v\right\|_{L^{2}(T)}^{2}\right.} \\
+ & \left.\sum_{e \in \mathcal{E}_{h}} h_{e}^{-1}\left\|\mathcal{P}_{0}^{e}\left[\partial_{\mathbf{e}_{i}} v\right]\right\|_{L^{2}(e)}^{2}\right] \quad \text { for } i=1,2,
\end{aligned}
$$

where $C>0$ is an $h$-independent constant, and $\mathcal{P}_{0}^{e}$ denotes the constant projection of $L^{2}(e)$ onto $\mathbb{P}_{0}(e)$.

In view of Lemma 3.2 and the definition of $V_{0}^{h}$, we have

$$
\mathcal{P}_{0}^{e}\left[\partial_{\mathbf{e}_{i}} v\right]=0 \quad \forall v \in V_{0}^{h}, \forall e \in \mathcal{E}_{h}, i=1,2 .
$$

Hence, by (3.8) we get

$$
\sum_{T \in \mathcal{T}_{h}}\left\|\partial_{\mathbf{e}_{i}} v\right\|_{L^{\infty}(T)}^{2} \leq C|\ln h| \sum_{T \in \mathcal{T}_{h}}\left\|D^{2} v\right\|_{L^{2}(T)}^{2} \quad \text { for } i=1,2 .
$$

Now, summing over all edges after adding (3.6) and (3.7), we obtain

$$
\begin{aligned}
& \sum_{e \in \mathcal{E}_{h}}\left(\left|\left\langle\left[\partial_{\bar{\tau} \bar{\tau}} v\right],\left\{\partial_{n} v\right\}\right\rangle_{e}\right|+\left|\left\langle\left[\partial_{\bar{\tau} \bar{n}} v\right],\left\{\partial_{\tau} v\right\}\right\rangle_{e}\right|\right) \\
& \quad \leq \frac{h|\ln h|}{\varepsilon} \sum_{e \in \mathcal{E}_{h}}\left(\left\|\left[\partial_{\bar{\tau} \bar{\tau}} v\right]\right\|_{L^{2}(e)}^{2}+\left\|\left[\partial_{\bar{\tau} \bar{n}} v\right]\right\|_{L^{2}(e)}^{2}\right)+\epsilon C \sum_{T \in \mathcal{T}_{h}}\left\|D^{2} v\right\|_{L^{2}(T)}^{2} .
\end{aligned}
$$

Step 3. Combining (3.5) and (3.9) gives us

$$
\begin{aligned}
\|v\|_{M}^{2} \geq \sum_{T \in \mathcal{T}_{h}}\left(\delta(1-\epsilon C)\left\|D^{2} v\right\|_{L^{2}(T)}^{2}+\|\nabla v\|_{L^{2}(T)}^{2}\right) \\
\quad+h|\ln h|\left(\gamma-\frac{\delta}{\epsilon}\right) \sum_{e \in \mathcal{E}_{h}}\left(\left\|\left[\partial_{\bar{\tau} \bar{\tau}} v\right]\right\|_{L^{2}(e)}^{2}+\left\|\left[\partial_{\bar{\tau} \bar{n}} v\right]\right\|_{L^{2}(e)}^{2}\right) .
\end{aligned}
$$

Choosing $\epsilon=\frac{1}{2 C}, \gamma_{0}=2 \delta C$, for $\gamma \geq \gamma_{0}$ we obtain

$$
\|v\|_{M}^{2} \geq \sum_{T \in \mathcal{T}_{h}}\left(\frac{\delta}{2}\left\|D^{2} v\right\|_{L^{2}(T)}^{2}+\|\nabla v\|_{L^{2}(T)}^{2}\right) \geq C \delta\|v\|_{2, h}^{2} .
$$

The proof is complete.

With Lemma 3.6 in hand, we are now able to show the first main result of this section.

Theorem 3.1. There exists a unique solution $u_{h}$ to (3.2). Furthermore, if $u \in$ $H^{3}(\Omega)$ and $\gamma \geq \gamma_{0}$, the following error estimates hold:

$$
\begin{aligned}
\left\|u-u_{h}\right\|_{M} & \leq h\left(C_{1}\|f\|_{L^{2}}+C_{2}\|u\|_{H^{3}}\right), \\
\left\|u-u_{h}\right\|_{2, h} & \leq \frac{C h}{\sqrt{\delta}}\left(C_{1}\|f\|_{L^{2}}+C_{2}\|u\|_{H^{3}}\right),
\end{aligned}
$$

where

$$
C_{1}=\frac{C M_{0}}{\sqrt{\delta}}, \quad C_{2}=C \sqrt{\delta+h^{2}+\gamma|\ln h|} .
$$


Proof. Since problem (3.2) is linear and in a finite dimensional setting, it suffices to show uniqueness. Thus, suppose $w \in V_{0}^{h}$ satisfies

$$
A_{h}^{\delta}(w, v)=0 \quad \forall v \in V_{0}^{h} .
$$

It follows that $w$ is piecewise constant on each $T \in \mathcal{T}_{h}$. By Corollary 3.1, we conclude $0=|w|_{1, h} \geq C\|w\|_{1, h}$, and hence $w \equiv 0$.

To show the error estimate (3.10), we use Strang's second lemma [7, 6] to get

$$
\left\|u-u_{h}\right\|_{M} \leq \inf _{v \in V_{0}^{h}}\|u-v\|_{M}+\sup _{0 \neq v \in V_{0}^{h}} \frac{\left|A_{h}^{\delta}(u, v)-(f, v)\right|}{\|v\|_{M}} .
$$

Using Lemma 3.4 ( $v_{0}$ is defined there), we obtain

$$
\begin{aligned}
(f, v)= & \left(\delta \square^{2} u-\Delta u, v_{0}\right)+\left(f, v-v_{0}\right) \\
= & \left(-\delta \bar{\nabla} \square u+\nabla u, \nabla v_{0}\right)+\left(f, v-v_{0}\right) \\
= & \sum_{T \in \mathcal{T}_{h}}\left(\delta(\square u, \square v)_{T}+(\nabla u, \nabla v)_{T}+\left(-\delta \bar{\nabla} \square u+\nabla u, \nabla\left(v_{0}-v\right)\right)_{T}\right. \\
& \left.\quad-\delta\left\langle\square u, \partial_{\bar{n}} v\right\rangle_{\partial T}\right)+\left(f, v-v_{0}\right) \\
= & \sum_{T \in \mathcal{T}_{h}}\left(\delta(\square u, \square v)_{T}+(\nabla u, \nabla v)_{T}+\left(-\delta \bar{\nabla} \square u+\nabla u, \nabla\left(v_{0}-v\right)\right)_{T}\right) \\
& \quad-\delta \sum_{e \in \mathcal{E}_{h}}\left\langle\square u,\left[\partial_{\bar{n}} v\right]\right\rangle_{e}+\left(f, v-v_{0}\right) \\
= & A_{h}^{\delta}(u, v)+\sum_{T \in \mathcal{T}_{h}}\left(-\delta \bar{\nabla} \square u+\nabla u, \nabla\left(v_{0}-v\right)\right)_{T} \\
& \quad-\delta \sum_{e \in \mathcal{E}_{h}}\left\langle\square u,\left[\partial_{\bar{n}} v\right]\right\rangle_{e}+\left(f, v-v_{0}\right) .
\end{aligned}
$$

Next, by Lemma 3.2, we have

$$
\begin{aligned}
\delta\left|\sum_{e \in \mathcal{E}_{h}}\left\langle\square u,\left[\partial_{\bar{n}} v\right]\right\rangle_{e}\right| & =\delta\left|\sum_{e \in \mathcal{E}_{h}}\left\langle\square u-\mathcal{P}_{0}^{e} \square u,\left[\partial_{\bar{n}} v\right]-\mathcal{P}_{0}^{e}\left[\partial_{\bar{n}} v\right]\right\rangle_{e}\right| \\
& \leq \delta C h\|\nabla \square u\|_{L^{2}}\|v\|_{2, h} .
\end{aligned}
$$

Also,

$$
\begin{aligned}
\left|\delta \sum_{T \in \mathcal{T}_{h}}\left(\bar{\nabla} \square u, \nabla\left(v_{0}-v\right)\right)_{T}\right| & =\left|\delta \sum_{T \in \mathcal{T}_{h}}\left(\nabla \square u, \bar{\nabla}\left(v_{0}-v\right)\right)_{T}\right| \\
& \leq \delta C h\|\nabla \square u\|_{L^{2}}\|v\|_{2, h},
\end{aligned}
$$

and by Lemma 3.4 we obtain

$$
\begin{aligned}
& \left|\sum_{T \in \mathcal{T}_{h}}\left(\nabla u, \nabla\left(v_{0}-v\right)\right)_{T}+\left(f, v-v_{0}\right)\right| \\
& \quad=\left|\sum_{T \in \mathcal{T}_{h}}\left(\Delta u, v-v_{0}\right)_{T}-\sum_{e \in \mathcal{E}_{h}}\left\langle\partial_{n} u,[v]\right\rangle_{e}+\left(f, v-v_{0}\right)\right| \\
& \quad \leq C\left(h^{\frac{3}{2}}\|\Delta u\|_{L^{2}}+h^{2}\|f\|_{L^{2}}\right)\|v\|_{2, h}+\sum_{e \in \mathcal{E}_{h}}\left\|\partial_{n} u\right\|_{L^{2}(e)}\|[v]\|_{L^{2}(e)} .
\end{aligned}
$$


By the Cauchy-Schwarz inequality, the trace/inverse inequality

$$
\left\|\partial_{n} u\right\|_{L^{2}(e)} \leq C h^{-\frac{1}{2}}\|u\|_{H^{2}(T)}
$$

(assuming $e$ is an edge of the element $T$, see (iii) of Lemma 2.1), and Lemma 3.3 we get

$$
\begin{aligned}
\sum_{e \in \mathcal{E}_{h}}\left\|\partial_{n} u\right\|_{L^{2}(e)}\|[v]\|_{L^{2}(e)} & \leq\left(\sum_{e \in \mathcal{E}_{h}}\left\|\partial_{n} u\right\|_{L^{2}(e)}^{2}\right)^{\frac{1}{2}}\left(\sum_{e \in \mathcal{E}_{h}}\|[v]\|_{L^{2}(e)}^{2}\right)^{\frac{1}{2}} \\
& \leq C\left(\sum_{T \in \mathcal{T}_{h}} h^{-1}\|u\|_{H^{2}(T)}^{2}\right)^{\frac{1}{2}}\left(\sum_{T \in \mathcal{T}_{h}} h^{3}\|v\|_{H^{2}(T)}^{2}\right)^{\frac{1}{2}} \\
& \leq C h\|u\|_{H^{2}}\|v\|_{2, h} \\
& \leq C h\|\Delta u\|_{L^{2}}\|v\|_{2, h} .
\end{aligned}
$$

Using these inequalities, the regularity result (2.3), and Lemma 3.6 it follows that

$$
\begin{aligned}
\left|A_{h}^{\delta}(u, v)-(f, v)\right| & \leq C h\left(\delta\|\nabla \square u\|_{L^{2}}+\|\Delta u\|_{L^{2}}+h\|f\|_{L^{2}}\right)\|v\|_{2, h}, \\
& \leq C h\left(\sqrt{\delta} M_{0}+M_{0}+h\right)\|f\|_{L^{2}}\|v\|_{2, h} \\
& \leq C M_{0} h\|f\|_{L^{2}}\|v\|_{2, h} \\
& \leq \frac{C M_{0} h}{\sqrt{\delta}}\|f\|_{L^{2}}\|v\|_{M} .
\end{aligned}
$$

Next, appealing to the inverse and trace inequalities, we have

$$
\begin{aligned}
\left\|u-\Pi_{h} u\right\|_{M}^{2}= & \sum_{T \in \mathcal{T}_{h}}\left(\delta\left\|\square\left(u-\Pi_{h} u\right)\right\|_{L^{2}(T)}^{2}+\left\|\nabla\left(u-\Pi_{h} u\right)\right\|_{L^{2}(T)}^{2}\right) \\
& +\sum_{e \in \mathcal{E}_{h}} \gamma h|\ln h|\left(\left\|\left[\partial_{\bar{\tau} \bar{\tau}}\left(u-\Pi_{h} u\right)\right]\right\|_{L^{2}(e)}^{2}+\left\|\left[\partial_{\bar{\tau} \bar{n}}\left(u-\Pi_{h} u\right)\right]\right\|_{L^{2}(e)}^{2}\right) \\
\leq & C h^{2}\left(\delta+h^{2}+\gamma|\ln h|\right)\|u\|_{H^{3}}^{2} .
\end{aligned}
$$

Thus,

$$
\inf _{v \in V_{0}^{h}}\|u-v\|_{M} \leq C h \sqrt{\delta+h^{2}+\gamma|\ln h|}\|u\|_{H^{3}} .
$$

Combining (3.12)-(3.14), we obtain

$$
\begin{aligned}
\left\|u-u_{h}\right\|_{M} & \leq C h\left(\frac{M_{0}}{\sqrt{\delta}}\|f\|_{L^{2}}+\sqrt{\delta+h^{2}+\gamma|\ln h|}\|u\|_{H^{3}}\right) \\
& \leq h\left(C_{1}\|f\|_{L^{2}}+C_{2}\|u\|_{H^{3}}\right) .
\end{aligned}
$$

To prove (3.11), we use the triangle inequality and Lemma 3.6

$$
\begin{aligned}
\left\|u-u_{h}\right\|_{2, h} & \leq\left\|u-\Pi_{h} u\right\|_{2, h}+\left\|\Pi_{h} u-u_{h}\right\|_{2, h} \\
& \leq C h\|u\|_{H^{3}}+\frac{C}{\sqrt{\delta}}\left\|\Pi_{h} u-u_{h}\right\|_{M} \\
& \leq C h\|u\|_{H^{3}}+\frac{C}{\sqrt{\delta}}\left(\left\|u-\Pi_{h} u\right\|_{M}+\left\|u-u_{h}\right\|_{M}\right) \\
& \leq \frac{C h}{\sqrt{\delta}}\left(C_{1}\|f\|_{L^{2}}+C_{2}\|u\|_{H^{3}}\right) .
\end{aligned}
$$


The second main theorem of this section, which is stated below, concerns the $H^{1}$-norm error estimate for the proposed Morley-type nonconforming method.

Theorem 3.2. In addition to the hypotheses of Theorem 3.1, assume $\Omega$ is convex. Then the following estimate holds:

$$
\left\|u-u_{h}\right\|_{1, h} \leq h^{2}\left(C_{3}\|f\|_{L^{2}}+C_{4}\|u\|_{H^{3}}\right),
$$

where

$$
\begin{aligned}
& C_{3}=C_{3}\left(\gamma, \delta, h, C_{E}\right)=C C_{E}\left(1+C_{2}^{2}+\frac{C_{2}(1+\delta|\ln h|)}{\sqrt{\delta}}\right), \\
& C_{4}=C_{4}\left(\gamma, \delta, h, C_{E}\right)=C C_{E}\left(h+C_{1} C_{2}+\frac{C_{1}(1+\delta|\ln h|)}{\sqrt{\delta}}\right),
\end{aligned}
$$

and $C_{E}$ is defined by (3.19).

Proof. The proof is quite technical, so we break it up into four steps.

Step 1 (A duality argument). Let $e_{h}=u-u_{h}, \rho_{h}=\Pi_{h} e_{h}=\Pi_{h} u-u_{h} \in V_{0}^{h}$ and $\rho_{0} \in H_{0}^{1}(\Omega)$ be as defined in Lemma 3.4. First, we state the following stability estimates:

$$
\begin{aligned}
\left\|\rho_{h}\right\|_{2, h} & \leq\left\|e_{h}\right\|_{2, h}+\left\|u-\Pi_{h} u\right\|_{2, h} \leq C\left\|e_{h}\right\|_{2, h}, \\
\left|\rho_{h}\right|_{1, h} & \leq\left|\rho_{h}-\rho_{0}\right|_{1, h}+\left\|\nabla \rho_{0}\right\|_{L^{2}} \\
& \leq h\left\|\rho_{h}\right\|_{2, h}+\left\|\nabla \rho_{0}\right\|_{L^{2}} \leq C h\left\|e_{h}\right\|_{2, h}+\left\|\nabla \rho_{0}\right\|_{L^{2}}, \\
\left\|\nabla \rho_{0}\right\|_{L^{2}} & \leq\left|\rho_{h}\right|_{1, h}+\left|\rho_{0}-\rho_{h}\right|_{1, h} \\
& \leq\left|\rho_{h}\right|_{1, h}+C h\left|\rho_{h}\right|_{2, h} \leq C\left|\rho_{h}\right|_{1, h} .
\end{aligned}
$$

Next, let $\varphi \in H^{3}(\Omega) \cap H_{0}^{2}(\Omega)$ be the solution to the following auxiliary problem:

$$
\begin{aligned}
\delta \Delta^{2} \varphi-\Delta \varphi & =-\Delta \rho_{0} & & \text { in } \Omega, \\
\varphi=\partial_{n} \varphi & =0 & & \text { on } \partial \Omega .
\end{aligned}
$$

Since $\rho_{0} \in H_{0}^{1}(\Omega)$ and $\Omega$ is convex, it follows from standard elliptic theory 9 that

$$
\|\varphi\|_{H^{3}} \leq C_{E}\left\|\nabla \rho_{0}\right\|_{L^{2}}
$$

Integrating by parts, we obtain

$$
\begin{aligned}
\left\|\nabla \rho_{0}\right\|_{L^{2}}^{2}= & \left(-\delta \nabla \Delta \varphi+\nabla \varphi, \nabla \rho_{0}\right) \\
= & \sum_{T \in \mathcal{T}_{h}}\left(\left(-\delta \nabla \Delta \varphi+\nabla \varphi, \nabla \rho_{h}\right)_{T}+\left(-\delta \nabla \Delta \varphi+\nabla \varphi, \nabla\left(\rho_{0}-\rho_{h}\right)\right)_{T}\right) \\
= & \sum_{T \in \mathcal{T}_{h}}\left(\delta\left(D^{2} \varphi, D^{2} \rho_{h}\right)_{T}+\left(\nabla \varphi, \nabla \rho_{h}\right)_{T}+\left(-\delta \nabla \Delta \varphi+\nabla \varphi, \nabla\left(\rho_{0}-\rho_{h}\right)\right)_{T}\right) \\
& \quad-\delta \sum_{e \in \mathcal{E}_{h}}\left(\left\langle\Delta \varphi-\partial_{\tau \tau} \varphi,\left[\partial_{n} \rho_{h}\right]\right\rangle_{e}+\left\langle\partial_{n \tau} \varphi,\left[\partial_{\tau} \rho_{h}\right]\right\rangle_{e}\right) .
\end{aligned}
$$

Applying Corollary 3.2 yields

$$
\begin{aligned}
\left(D^{2} \varphi, D^{2} \rho_{h}\right)_{T} & =\left(\square \varphi, \square \rho_{h}\right)_{T}+Q_{T}\left(\Pi_{h} \varphi, \rho_{h}\right)+Q_{T}\left(\varphi-\Pi_{h} \varphi, \rho_{h}\right) \\
& =\left(\square \varphi, \square \rho_{h}\right)_{T}+S_{T}\left(\varphi, \rho_{h}\right)+S_{T}\left(\Pi_{h} \varphi-\varphi, \rho_{h}\right)+Q_{T}\left(\varphi-\Pi_{h} \varphi, \rho_{h}\right),
\end{aligned}
$$


and hence, we obtain the following identity:

$$
\begin{aligned}
\left\|\nabla \rho_{0}\right\|_{L^{2}}^{2}=A_{h}^{\delta}\left(\varphi, \rho_{h}\right)+\sum_{T \in \mathcal{T}_{h}}\left(-\delta \nabla \Delta \varphi+\nabla \varphi, \nabla\left(\rho_{0}-\rho_{h}\right)\right)_{T} \\
\quad-\delta \sum_{e \in \mathcal{E}_{h}}\left(\left\langle\Delta \varphi-\partial_{\tau \tau} \varphi,\left[\partial_{n} \rho_{h}\right]\right\rangle_{e}+\left\langle\partial_{n \tau} \varphi,\left[\partial_{\tau} \rho_{h}\right]\right\rangle_{e}\right) \\
+\delta\left(S\left(\varphi, \rho_{h}\right)+S\left(\Pi_{h} \varphi-\varphi, \rho_{h}\right)+Q\left(\varphi-\Pi_{h} \varphi, \rho_{h}\right)\right) .
\end{aligned}
$$

Step 2 (Bounding the last six terms in (3.20) ). First, using standard interpolation results and (3.16) we get

$$
Q\left(\varphi-\Pi_{h} \varphi, \rho_{h}\right) \leq C\left|\varphi-\Pi_{h} \varphi\right|_{2, h}\left|\rho_{h}\right|_{2, h} \leq C h\|\varphi\|_{H^{3}}\left\|e_{h}\right\|_{2, h} .
$$

Next, using the trace inequality we have for any $T \in \mathcal{T}_{h}$,

$$
\begin{aligned}
S_{T}\left(\Pi_{h} \varphi-\varphi, \rho_{h}\right) & =\left\langle\partial_{\bar{\tau} \bar{\tau}}\left(\Pi_{h} \varphi-\varphi\right), \partial_{n} \rho_{h}\right\rangle_{\partial T}-\left\langle\partial_{\bar{\tau} \bar{n}}\left(\Pi_{h} \varphi-\varphi\right), \partial_{\tau} \rho_{h}\right\rangle_{\partial T} \\
& \leq\left\|\partial_{\bar{\tau} \bar{\tau}}\left(\Pi_{h} \varphi-\varphi\right)\right\|_{L^{2}(\partial T)}\left\|\partial_{n} \rho_{h}\right\|_{L^{2}(\partial T)} \\
& \quad+\left\|\partial_{\bar{\tau} \bar{n}}\left(\Pi_{h} \varphi-\varphi\right)\right\|_{L^{2}(\partial T)}\left\|\partial_{\tau} \rho_{h}\right\|_{L^{2}(\partial T)} \\
& \leq C h^{\frac{1}{2}}\|\varphi\|_{H^{3}(T)}\left\|\nabla \rho_{h}\right\|_{L^{2}(\partial T)} \\
\leq & \leq C h\|\varphi\|_{H^{3}(T)}\left\|\nabla \rho_{h}\right\|_{L^{\infty}(T)} .
\end{aligned}
$$

Therefore, using (3.17) and a similar technique as that found in Step 2 of the proof of Lemma 3.6, we have

$$
\begin{aligned}
S\left(\Pi_{h} \varphi-\varphi, \rho_{h}\right) & =\sum_{T \in \mathcal{T}_{h}} S_{T}\left(\Pi_{h} \varphi-\varphi, \rho_{h}\right) \\
& \leq C h|\ln h|\|\varphi\|_{H^{3}}\left\|\rho_{h}\right\|_{2, h} \\
& \leq C h|\ln h|\|\varphi\|_{H^{3}}\left\|e_{h}\right\|_{2, h} .
\end{aligned}
$$

Next, using Lemma 3.2 and (3.16)

$$
\begin{aligned}
S\left(\varphi, \rho_{h}\right)= & \sum_{e \in \mathcal{E}_{h}}\left(\left\langle\partial_{\bar{\tau} \bar{\tau}} \varphi,\left[\partial_{n} \rho_{h}\right]\right\rangle_{e}-\left\langle\partial_{\bar{\tau} \bar{n}} \varphi,\left[\partial_{\tau} \rho_{h}\right]\right\rangle_{e}\right) \\
= & \sum_{e \in \mathcal{E}_{h}}\left(\left\langle\partial_{\bar{\tau} \bar{\tau}} \varphi-\mathcal{P}_{0}^{e}\left(\partial_{\bar{\tau} \bar{\tau}} \varphi\right),\left[\partial_{n} \rho_{h}\right]-\mathcal{P}_{0}^{e}\left(\left[\partial_{n} \rho_{h}\right]\right)\right\rangle_{e}\right. \\
& \left.\quad-\left\langle\partial_{\bar{\tau} \bar{n}} \varphi-\mathcal{P}_{0}^{e}\left(\partial_{\bar{\tau} \bar{n}} \varphi\right),\left[\partial_{\tau} \rho_{h}\right]-\mathcal{P}_{0}^{e}\left(\left[\partial_{\tau} \rho_{h}\right]\right)\right\rangle_{e}\right) \\
\leq & C h\|\varphi\|_{H^{3}}\left\|\rho_{h}\right\|_{2, h} \\
\leq & C h\|\varphi\|_{H^{3}}\left\|e_{h}\right\|_{2, h},
\end{aligned}
$$

Similarly, we conclude

$$
\sum_{e \in \mathcal{E}_{h}}\left(\left\langle\Delta \varphi-\partial_{\tau \tau} \varphi,\left[\partial_{n} \rho_{h}\right]\right\rangle_{e}+\left\langle\partial_{n \tau} \varphi,\left[\partial_{\tau} \rho_{h}\right]\right\rangle_{e}\right) \leq C h\|\varphi\|_{H^{3}}\left\|e_{h}\right\|_{2, h},
$$

and using Lemma 3.4, we obtain

$$
\begin{aligned}
\sum_{T \in \mathcal{T}_{h}}\left(-\delta \nabla \Delta \varphi+\nabla \varphi, \nabla\left(\rho_{0}-\rho_{h}\right)\right)_{T} & \leq C\|\varphi\|_{H^{3}}\left\|\rho_{0}-\rho_{h}\right\|_{1, h} \\
& \leq C h\|\varphi\|_{H^{3}}\left\|e_{h}\right\|_{2, h} .
\end{aligned}
$$


Applying bounds (3.21)-(3.25) to (3.20), we have

$$
\left\|\nabla \rho_{0}\right\|_{L^{2}}^{2} \leq A_{h}^{\delta}\left(\varphi, \rho_{h}\right)+C h(1+\delta|\ln h|)\left\|e_{h}\right\|_{2, h}\|\varphi\|_{H^{3}} .
$$

Step 3 (Bounding $A_{h}^{\delta}\left(\varphi, \rho_{h}\right)$ ). To bound $A_{h}^{\delta}\left(\varphi, \rho_{h}\right)$, we write

$$
A_{h}^{\delta}\left(\varphi, \rho_{h}\right)=A_{h}^{\delta}\left(e_{h}, \varphi-\Pi_{h} \varphi\right)+A_{h}^{\delta}\left(e_{h}, \Pi_{h} \varphi\right)+A_{h}^{\delta}\left(\varphi, \Pi_{h} u-u\right) .
$$

Bounding the third term in (3.27) we get

$$
\begin{aligned}
A_{h}^{\delta}\left(\varphi, \Pi_{h} u-u\right)=\sum_{T \in \mathcal{T}_{h}}\left(\delta\left(\square \varphi, \square\left(\Pi_{h} u-u\right)\right)_{T}+\left(\nabla \varphi, \nabla\left(\Pi_{h} u-u\right)\right)_{T}\right) \\
=\sum_{T \in \mathcal{T}_{h}}\left(-\delta\left(\nabla \square \varphi, \bar{\nabla}\left(\Pi_{h} u-u\right)\right)_{T}+\left(\nabla \varphi, \nabla\left(\Pi_{h} u-u\right)\right)_{T}\right) \\
\quad+\sum_{e \in \mathcal{E}_{h}} \delta\left\langle\square \varphi-\mathcal{P}_{0}^{e}(\square \varphi),\left[\partial_{\bar{n}}\left(\Pi_{h} u-u\right)\right]-\mathcal{P}_{0}^{e}\left(\left[\partial_{\bar{n}}\left(\Pi_{h} u-u\right)\right]\right)\right\rangle_{e} \\
\leq C h^{2}\|\varphi\|_{H^{3}}\|u\|_{H^{3}} .
\end{aligned}
$$

To bound the second term in (3.27), we have

$$
\begin{aligned}
& A_{h}^{\delta}\left(e_{h}, \Pi_{h} \varphi\right)=A_{h}^{\delta}\left(u, \Pi_{h} \varphi\right)-(f, \varphi)-\left(f, \Pi_{h} \varphi-\varphi\right) \\
& =\sum_{T \in \mathcal{T}_{h}}\left(-\delta \bar{\nabla} \square u+\nabla u, \nabla\left(\Pi_{h} \varphi-\varphi\right)\right)_{T}-\left(f, \Pi_{h} \varphi-\varphi\right) \\
& \quad+\delta \sum_{e \in \mathcal{E}_{h}}\left\langle\square u-\mathcal{P}_{0}^{e}(\square u),\left[\partial_{\bar{n}}\left(\Pi_{h} \varphi-\varphi\right)\right]-\mathcal{P}_{0}^{e}\left[\partial_{\bar{n}}\left(\Pi_{h} \varphi-\varphi\right)\right]\right\rangle_{e} \\
& \leq C h^{2}\left(\|u\|_{H^{3}}+h\|f\|_{L^{2}}\right)\|\varphi\|_{H^{3}} .
\end{aligned}
$$

We bound the first term in (3.27) as follows:

$$
\begin{aligned}
A_{h}^{\delta}\left(e_{h}, \varphi-\Pi_{h} \varphi\right) & \leq\left\|e_{h}\right\|_{M}\left\|\varphi-\Pi_{h} \varphi\right\|_{M} \\
& \leq C C_{2} h\|\varphi\|_{H^{3}}\left\|e_{h}\right\|_{M} .
\end{aligned}
$$

Combining (3.27)-(3.30), we obtain

$$
A_{h}^{\delta}\left(\varphi, \rho_{h}\right) \leq C h\left(h\|u\|_{H^{3}}+h^{2}\|f\|_{L^{2}}+C_{2}\left\|e_{h}\right\|_{M}\right)\|\varphi\|_{H^{3}} .
$$

Step 4 (Finishing up). Using bounds (3.26) and (3.31), and the regularity result (3.19), we conclude

$$
\begin{gathered}
\left\|\nabla \rho_{0}\right\|_{L^{2}} \leq C C_{E}\left(h^{2}\|u\|_{H^{3}}+h^{3}\|f\|_{L^{2}}+C_{2} h\left\|e_{h}\right\|_{M}+h(1+\delta|\ln h|)\left\|e_{h}\right\|_{2, h}\right) \\
\leq C C_{E} h^{2}\left[\left(1+C_{2}^{2}+\frac{C_{2}(1+\delta|\ln h|)}{\sqrt{\delta}}\right)\|u\|_{H^{3}}\right. \\
\left.+\left(h+C_{1} C_{2}+\frac{C_{1}(1+\delta|\ln h|)}{\sqrt{\delta}}\right)\|f\|_{L^{2}}\right] .
\end{gathered}
$$


Finally, using the stability result (3.18), we have

$$
\begin{aligned}
\left|u-u_{h}\right|_{1, h} \leq & \left|u-\Pi_{h} u\right|_{1, h}+\left|\rho_{h}\right|_{1, h} \\
\leq & C C_{E} h^{2}\left[\left(1+C_{2}^{2}+\frac{C_{2}(1+\delta|\ln h|)}{\sqrt{\delta}}\right)\|u\|_{H^{3}}\right. \\
& \left.\quad+\left(h+C_{1} C_{2}+\frac{C_{1}(1+\delta|\ln h|)}{\sqrt{\delta}}\right)\|f\|_{L^{2}}\right] \\
& \leq h^{2}\left(C_{3}\|f\|_{L^{2}}+C_{4}\|u\|_{H^{3}}\right) .
\end{aligned}
$$

The proof is complete.

We conclude this section by remarking that all results of this section are still valid if the Morley-type nonconforming element $S_{2}^{h}$ is replaced by the original Morley element (cf. [17, 20]) but making no change to the formulation of the method.

\section{Interior PENALTy Discontinuous GALERKIN METHOdS}

In the previous section we constructed and analyzed a quadratic Morley-type nonconforming finite element method for problem (1.1)-(1.2). As in the case of conforming finite elements [13, the construction of the Morley-type element is only possible on some special meshes (it is interesting to note that the mesh constraints for the conforming elements and for the nonconforming finite element are "orthogonal" to each other). Moreover, in order to ensure the convergence of the Morley-type nonconforming finite element method, two (super)penalized jump terms must be introduced in the mesh-dependent bilinear form $A_{h}^{\delta}(\cdot, \cdot)$. These jump terms are not only critically used in the convergence proof but also certified by numerical experiments (see Section 5) to be indispensable for the convergence of the method.

To avoid the mesh constraints imposed by both conforming and nonconforming finite element methods, and also considering the fact that interior penalty terms must be used in the nonconforming method, it is natural to go one step further and develop interior penalty discontinuous Galerkin (DG) methods (cf. [10, 3, 23, 1]). This indeed is the main goal of this section.

In this section, we develop a family of interior penalty DG methods for problem (1.1)-11.2). Our methods are closely related to the DG methods introduced by Baker [3] (also see [12) for the biharmonic problem. As it is now well known (cf. 2, 19 and the references therein), DG methods use trial and test spaces consisting of totally discontinuous polynomials, and as a result, the weak formulation naturally include jump and average terms across element edges/faces, and penalty terms are introduced to control the discontinuity between adjacent elements. DG methods enjoy a number of advantages over (conforming and nonconforming) finite element methods. This is especially true for fourth-order problems such as (1.1)-(1.2) and the biharmonic problem, which are difficult and delicate to solve by finite element methods.

4.1. Formulation of interior penalty DG methods. To formulate interior penalty DG methods for problem (1.1)-(1.2), we introduce some additional notation. Let $\mathcal{T}_{h}$ be a locally quasi-uniform triangulation of $\Omega$ with $h_{T}=\operatorname{diam}(T)$ and $h=\max _{T \in \mathcal{T}_{h}} h_{T}$. The notations $\mathcal{E}_{h}^{I}, \mathcal{E}_{h}^{B}, \mathcal{E}_{h},[v]$, and $\{v\}$ are the same as in 
Section 3. We also define

$$
W_{r}^{h}=\prod_{T \in \mathcal{T}_{h}} \mathbb{P}_{r}(T), \quad \stackrel{\circ}{W}_{r}^{h}=W_{r}^{h} \cap H_{0}^{1}(\Omega), \quad H^{s}\left(\mathcal{T}_{h}\right)=\prod_{T \in \mathcal{T}_{h}} H^{s}(T),
$$

where $r \geq 2$ which will be assumed in the rest of this section.

For $T \in \mathcal{T}_{h}$, it is easy to check that the following Green's identities hold for the d'Alembertian $\square$ and the bi-wave operator $\square^{2}$ :

$$
\begin{aligned}
\int_{T} \square v w d x d y & =\int_{\partial T} \partial_{\bar{n}} v w d s-\int_{T} \nabla v \cdot \bar{\nabla} w d x d y \\
\int_{T} \square^{2} v w d x d y & =\int_{\partial T}\left(\partial_{\bar{n}} \square v\right) w d s-\int_{\partial T} \square v \partial_{\bar{n}} w d s+\int_{T} \square v \square w d x d y .
\end{aligned}
$$

The second identity together with the elementary algebraic identity,

$$
a^{+} b^{+}-a^{-} b^{-}=[a]\{b\}+\{a\}[b],
$$

motivates us to introduce the following mesh-dependent bilinear form on the product space $H^{4}\left(\mathcal{T}_{h}\right) \times H^{4}\left(\mathcal{T}_{h}\right)$ :

$$
\begin{aligned}
a_{h}^{\delta}(v, w):= & \sum_{T \in \mathcal{T}_{h}}\left(\delta(\square v, \square w)_{T}+(\nabla v, \nabla w)_{T}\right)+\sum_{e \in \mathcal{E}_{h}}\left(\left\langle\delta\left\{\partial_{\bar{n}} \square v\right\}-\left\{\partial_{n} v\right\},[w]\right\rangle_{e}\right. \\
& -\delta\left\langle\{\square v\},\left[\partial_{\bar{n}} w\right]\right\rangle_{e}+\left\langle\delta\left\{\partial_{\bar{n}} \square w\right\}-\left\{\partial_{n} w\right\},[v]\right\rangle_{e}-\delta\left\langle\{\square w\},\left[\partial_{\bar{n}} v\right]\right\rangle_{e} \\
& \left.+\gamma h_{e}^{-1}\left\langle\left[\partial_{\bar{n}} v\right],\left[\partial_{\bar{n}} w\right]\right\rangle_{e}+\gamma h_{e}^{-3}\langle[v],[w]\rangle_{e}\right) \quad \forall v, w \in H^{4}\left(\mathcal{T}_{h}\right) .
\end{aligned}
$$

As in the previous section, $\gamma$ is a positive constant independent of $h$ and the terms involving $\gamma$ are the so-called penalty terms.

The bilinear form $a_{h}^{\delta}(\cdot, \cdot)$ induces the following norm on $H^{4}\left(\mathcal{T}_{h}\right)$ :

$$
\begin{aligned}
\|v\|_{E}^{2}:= & \sum_{T \in \mathcal{T}_{h}}\left(\delta\|\square v\|_{L^{2}(T)}^{2}+\|\nabla v\|_{L^{2}(T)}^{2}\right) \\
& +\sum_{e \in \mathcal{E}_{h}}\left(\gamma h_{e}^{-3}\|[v]\|_{L^{2}(e)}^{2}+\gamma h_{e}^{-1}\left\|\left[\partial_{\bar{n}} v\right]\right\|_{L^{2}(e)}^{2}\right. \\
& \left.+\delta h_{e}\|\{\square v\}\|_{L^{2}(e)}^{2}+\delta h_{e}^{3}\left\|\left\{\partial_{\bar{n}} \square v\right\}\right\|_{L^{2}(e)}^{2}+h_{e}\left\|\left\{\partial_{n} v\right\}\right\|_{L^{2}(e)}^{2}\right) .
\end{aligned}
$$

We also define the following alternative norm which will be used later in the paper:

$$
\begin{aligned}
\|v\|_{\tilde{E}}^{2}:= & \sum_{T \in \mathcal{T}_{h}}\left(\delta\|\square v\|_{L^{2}(T)}^{2}+\|\nabla v\|_{L^{2}(T)}^{2}\right)+\sum_{e \in \mathcal{E}_{h}}\left(\gamma h_{e}^{-3}\|[v]\|_{L^{2}(e)}^{2}\right. \\
& \left.+\gamma h_{e}^{-1}\left\|\left[\partial_{\bar{n}} v\right]\right\|_{L^{2}(e)}^{2}+\delta h_{e}\|\{\square v\}\|_{L^{2}(e)}^{2}+h_{e}\left\|\left\{\partial_{n} v\right\}\right\|_{L^{2}(e)}^{2}\right) .
\end{aligned}
$$

We now define a weak formulation of (1.1)-(1.2) as seeking $u \in H^{4}\left(\mathcal{T}_{h}\right) \cap V$ such that

$$
a_{h}^{\gamma}(u, v)=(f, v) \quad \forall v \in H^{4}\left(\mathcal{T}_{h}\right) \cap V .
$$

Remark 4.1. One may easily verify that the formulation (4.3) is consistent. That is, if $u \in H^{4}(\Omega) \cap V$ is the solution to (1.1)-(1.2), then $u$ satisfies (4.3). Conversely, if $u \in H^{4}(\Omega) \cap V_{0}$ solves (4.3), then $u$ is the unique solution to (1.1)-(1.2).

Based on (4.3), we then define our interior penalty discontinuous Galerkin methods as to find $u_{h} \in W_{r}^{h}$ such that

$$
a_{h}^{\gamma}\left(u_{h}, v\right)=(f, v) \quad \forall v \in W_{r}^{h},
$$


We end this subsection by stating a lemma which concerns the approximation properties of the finite element spaces $W_{r}^{h}$. We omit the proof to save space and refer the reader to 3 for the proof of similar results.

Lemma 4.1. For any $v \in H^{s}(\Omega)(s \geq 4)$, there exists $\tilde{v} \in W_{r}^{h}$ such that

$$
\begin{array}{rlrl}
\|v-\tilde{v}\|_{E} & \leq C\left(\sum_{T \in \mathcal{T}_{h}} h_{T}^{2 \ell-4}\left(\delta+\gamma+h_{T}^{2}\right)\|v\|_{H^{\ell}(T)}^{2}\right)^{\frac{1}{2}} & & \\
& \leq C h^{\ell-2}(\sqrt{\gamma+\delta}+h)\|v\|_{H^{\ell}}, & r \geq 3, \\
\|v-\tilde{v}\|_{E} & \leq C\left(\sum_{T \in \mathcal{T}_{h}} h_{T}^{2}\left(\delta+\gamma+h_{T}^{2}\right)\|v\|_{H^{4}(T)}^{2}\right)^{\frac{1}{2}} & \\
& \leq C h(\sqrt{\gamma+\delta}+h)\|v\|_{H^{4}}, & & r=2 .
\end{array}
$$

Moreover, if $v \in H^{s}(\Omega)(s \geq 3)$, there exists $\tilde{v} \in W_{r}^{h}$ such that

$$
\begin{aligned}
\|v-\tilde{v}\|_{\tilde{E}} & \leq C\left(\sum_{T \in \mathcal{T}_{h}} h_{T}^{2 \ell-4}\left(\delta+\gamma+h_{T}^{2}\right)\|v\|_{H^{\ell}(T)}^{2}\right)^{\frac{1}{2}} & \\
& \leq C h^{\ell-2}(\sqrt{\gamma+\delta}+h)\|v\|_{H^{\ell}}, & r \geq 2 .
\end{aligned}
$$

Where $\ell=\min \{s, r+1\}$.

4.2. Convergence analysis of interior penalty DG methods. The following lemma ensures that the bilinear form $a_{h}^{\gamma}(\cdot, \cdot)$ is continuous and coercive on the finite element space $W_{r}^{h}$.

Lemma 4.2. The following inequality holds:

$$
\left|a_{h}^{\delta}(v, w)\right| \leq 2\|v\|_{E}\|w\|_{E} \quad \forall v, w \in H^{4}\left(\mathcal{T}_{h}\right) .
$$

Furthermore, there exists a positive constant $\gamma_{0}=O\left(\delta+h^{2}\right)$ such that for $\gamma \geq \gamma_{0}$, we get

$$
a_{h}^{\delta}(v, v) \geq C\|v\|_{E}^{2} \quad \forall v \in W_{r}^{h} .
$$

Proof. The proof is similar to the one found in [3, Propositions 5.1 and 5.2] (also see [19]). Clearly (4.5) is a direct consequence of the Cauchy-Schwarz inequality, so we only give a detailed proof of (4.6).

First, for $v \in W_{r}^{h}$ we have using the Cauchy-Schwarz and inverse inequalities

$$
\begin{aligned}
\|v\|_{E}^{2} \leq & C \sum_{T \in \mathcal{T}_{h}}\left(\delta\|\square v\|_{L^{2}(T)}^{2}+\|\nabla v\|_{L^{2}(T)}^{2}\right) \\
& +C \sum_{e \in \mathcal{E}_{h}}\left(\gamma h_{e}^{-3}\|[v]\|_{L^{2}(e)}^{2}+\gamma h_{e}^{-1}\left\|\left[\partial_{\bar{n}} v\right]\right\|_{L^{2}(e)}^{2}\right) .
\end{aligned}
$$

Next, by definition we have

$$
\begin{aligned}
a_{h}^{\gamma}(v, v) \geq & \sum_{T \in \mathcal{T}_{h}}\left(\delta\|\square v\|_{L^{2}(T)}^{2}+\|\nabla v\|_{L^{2}(T)}^{2}\right) \\
& +\sum_{e \in \mathcal{E}_{h}}\left(\gamma h_{e}^{-3}\|[v]\|_{L^{2}(e)}^{2}+\gamma h_{e}^{-1}\left\|\left[\partial_{\bar{n}} v\right]\right\|_{L^{2}(e)}^{2}\right. \\
& \left.\quad-2\left|\left\langle\delta\left\{\partial_{\bar{n}} \square v\right\}-\left\{\partial_{n} v\right\},[v]\right\rangle_{e}\right|-2 \delta\left|\left\langle\{\square v\},\left[\partial_{\bar{n}} v\right]\right\rangle_{e}\right|\right) .
\end{aligned}
$$


Using the trace and inverse inequalities yields for $e=T_{1} \cap T_{2} \in \mathcal{E}_{h}^{I}$,

$$
\begin{gathered}
\left|\left\langle\left\{\partial_{\bar{n}} \square v\right\},[v]\right\rangle_{e}\right| \leq C\left(\|\square v\|_{L^{2}\left(T_{1}\right)}+\|\square v\|_{L^{2}\left(T_{2}\right)}\right) h_{e}^{-\frac{3}{2}}\|[v]\|_{L^{2}(e)}, \\
\left|\left\langle\{\square v\},\left[\partial_{\bar{n}} v\right]\right\rangle_{e}\right| \leq C\left(\|\square v\|_{L^{2}\left(T_{1}\right)}+\|\square v\|_{L^{2}\left(T_{2}\right)}\right) h_{e}^{-\frac{1}{2}}\left\|\left[\partial_{\bar{n}} v\right]\right\|_{L^{2}(e)}, \\
\left|\left\langle\left\{\partial_{n} v\right\},[v]\right\rangle_{e}\right| \leq C\left(\|\nabla v\|_{L^{2}\left(T_{1}\right)}+\|\nabla v\|_{L^{2}\left(T_{2}\right)}\right) h_{e}^{-\frac{1}{2}}\|[v]\|_{L^{2}(e)} .
\end{gathered}
$$

Similar inequalities hold on $e \in \mathcal{E}_{h}^{B}$. Thus,

$$
\begin{aligned}
\sum_{e \in \mathcal{E}_{h}} \delta\left|\left\langle\left\{\partial_{\bar{n}} \square v\right\},[v]\right\rangle_{e}\right| & \leq \delta C\left(\sum_{T \in \mathcal{T}_{h}}\|\square v\|_{L^{2}(T)}^{2}\right)^{\frac{1}{2}}\left(\sum_{e \in \mathcal{E}_{h}} h_{e}^{-3}\|[v]\|_{L^{2}(e)}^{2}\right)^{\frac{1}{2}} \\
& \leq \frac{\delta}{8} \sum_{T \in \mathcal{T}_{h}}\|\square v\|_{L^{2}(T)}^{2}+\delta C \sum_{e \in \mathcal{E}_{h}} h_{e}^{-3}\|[v]\|_{L^{2}(e)}^{2}, \\
\sum_{e \in \mathcal{E}_{h}} \delta\left|\left\langle\{\square v\},\left[\partial_{\bar{n}} v\right]\right\rangle_{e}\right| & \leq \delta C\left(\sum_{T \in \mathcal{T}_{h}}\|\square v\|_{L^{2}(T)}^{2}\right)^{\frac{1}{2}}\left(\sum_{e \in \mathcal{E}_{h}} h_{e}^{-1}\left\|\left[\partial_{\bar{n}} v\right]\right\|_{L^{2}(e)}^{2}\right)^{\frac{1}{2}}, \\
& \leq \frac{\delta}{8} \sum_{T \in \mathcal{T}_{h}}\|\square v\|_{L^{2}(T)}^{2}+\delta C \sum_{e \in \mathcal{E}_{h}} h_{e}^{-1}\left\|\left[\partial_{\bar{n}} v\right]\right\|_{L^{2}(e)}^{2}, \\
\sum_{e \in \mathcal{E}_{h}}\left|\left\langle\left\{\partial_{n} v\right\},\left[v_{h}\right]\right\rangle_{e}\right| & \leq C\left(\sum_{T \in \mathcal{T}_{h}}\|\nabla v\|_{L^{2}(T)}^{2}\right)^{\frac{1}{2}}\left(\sum_{e \in \mathcal{E}_{h}} h_{e}^{-1}\|[v]\|_{L^{2}(e)}^{2}\right)^{\frac{1}{2}} \\
& \leq \frac{1}{4} \sum_{T \in \mathcal{T}_{h}}\|\nabla v\|_{L^{2}(T)}^{2}+C \sum_{e \in \mathcal{E}_{h}} h_{e}^{-1}\|[v]\|_{L^{2}(e)}^{2} .
\end{aligned}
$$

Combining these estimates with (4.8) we obtain

$$
\begin{aligned}
& a_{h}^{\delta}(v, v) \geq \frac{1}{2} \sum_{T \in \mathcal{T}_{h}}\left(\delta\|\square v\|_{L^{2}(T)}^{2}+\|\nabla v\|_{L^{2}(T)}^{2}\right) \\
& \quad+\sum_{e \in \mathcal{E}_{h}}\left(h_{e}^{-3}\left(\gamma-C\left(\delta+h_{e}^{2}\right)\right)\|[v]\|_{L^{2}(e)}^{2}\right. \\
&\left.\quad+h_{e}^{-1}(\gamma-\delta C)\left\|\left[\partial_{\bar{n}} v\right]\right\|_{L^{2}(e)}^{2}\right) .
\end{aligned}
$$

Choosing $\gamma_{0}=C\left(\delta+h^{2}\right)$ and using (4.7), we have for $\gamma \geq \gamma_{0}$,

$$
a_{h}^{\delta}(v, v) \geq C\|v\|_{E}^{2} .
$$

An immediate consequence of the above lemma is the following existence and uniqueness theorem.

Theorem 4.1. The discrete problem (4.4) has a unique solution for $\gamma \geq \gamma_{0}$.

We now are ready to state and prove one of the main theorems of this section.

Theorem 4.2. Let $\gamma \geq \gamma_{0}$ and suppose $u \in H^{s}(\Omega)(s \geq 4)$ solves (1.1)-(1.2). Then the following error estimates hold:

$$
\begin{aligned}
\left\|u-u_{h}\right\|_{E} \leq C h^{\ell-2}(\sqrt{\gamma+\delta}+h)\|u\|_{H^{\ell}}, & r & \geq 3, \\
\left\|u-u_{h}\right\|_{E} \leq C h(\sqrt{\gamma+\delta}+h)\|u\|_{H^{4}}, & r & =2 .
\end{aligned}
$$

where $\ell=\min \{r+1, s\}$, and $C$ is independent of $h, \gamma$, and $\delta$. 
Proof. Using the consistency, coercivity, and continuity of the bilinear form $a_{h}^{\delta}$, we have for $\gamma \geq \gamma_{0}$ and $v \in W_{r}^{h}$,

$$
\begin{aligned}
C\left\|u_{h}-v\right\|_{E}^{2} & \leq a_{h}^{\delta}\left(u_{h}-v, u_{h}-v\right)=a_{h}^{\delta}\left(u-v, u_{h}-v\right) \\
& \leq 2\|u-v\|_{E}\left\|u_{h}-v\right\|_{E},
\end{aligned}
$$

and therefore

$$
\left\|u-u_{h}\right\|_{E} \leq\|u-v\|_{E}+\left\|u_{h}-v\right\|_{E} \leq C\|u-v\|_{E} .
$$

The error estimates (4.9)-(4.10) then follow directly from Lemma 4.1

Next, we derive error estimates when the solution only belongs to $H^{3}(\Omega)$. To conclude, we need the following technical lemma which is concerned with how well a function in $W_{r}^{h}$ can be approximated by continuous functions. A proof of the lemma can be found in [15].

Lemma 4.3. For any $v \in W_{r}^{h}$, there exists $E_{h} v \in \stackrel{\circ}{W}_{r}^{h}$ such that

$$
\begin{aligned}
& \left\|v-E_{h} v\right\|_{L^{2}}^{2} \leq C \sum_{e \in \mathcal{E}_{h}^{I}} h_{e}\|[v]\|_{L^{2}(e)}^{2}+\sum_{e \in \mathcal{E}_{h}^{B}} h_{e}\|v\|_{L^{2}(e)}^{2}, \\
& \left|v-E_{h} v\right|_{1, h}^{2} \leq C \sum_{e \in \mathcal{E}_{h}^{I}} h_{e}^{-1}\|[v]\|_{L^{2}(e)}^{2}+\sum_{e \in \mathcal{E}_{h}^{B}} h_{e}^{-1}\|v\|_{L^{2}(e)}^{2} .
\end{aligned}
$$

Theorem 4.3. Suppose $u \in H^{3}(\Omega)$ is the unique solution to (1.1)-(1.2). Then for $\gamma \geq \gamma_{0}, r=2$, the following error estimate holds:

$$
\left\|u-u_{h}\right\|_{\tilde{E}} \leq C h\left\{(\sqrt{\gamma+\delta}+h)\|u\|_{H^{3}}+M_{0}(\sqrt{\delta}+h)\|f\|_{L^{2}}\right\} .
$$

Proof. We note that in the case $r=2, u_{h}$ satisfies

$$
\tilde{a}_{h}^{\delta}\left(u_{h}, v\right)=(f, v) \quad \forall v \in W_{2}^{h},
$$

where

$$
\begin{aligned}
\tilde{a}_{h}^{\delta}(v, w):= & \sum_{T \in \mathcal{T}_{h}}\left(\delta(\square v, \square w)_{T}+(\nabla v, \nabla w)_{T}\right)-\sum_{e \in \mathcal{E}_{h}}\left(\left\langle\left\{\partial_{n} v\right\},[w]\right\rangle_{e}\right. \\
& +\delta\left\langle\{\square v\},\left[\partial_{\bar{n}} w\right]\right\rangle_{e}+\left\langle\left\{\partial_{n} w\right\},[v]\right\rangle_{e}+\delta\left\langle\{\square w\},\left[\partial_{\bar{n}} v\right]\right\rangle_{e} \\
& \left.-\gamma h_{e}^{-1}\left\langle\left[\partial_{\bar{n}} v\right],\left[\partial_{\bar{n}} w\right]\right\rangle_{e}-\gamma h_{e}^{-3}\langle[v],[w]\rangle_{e}\right) .
\end{aligned}
$$

We also have for $\gamma \geq \gamma_{0}$,

$$
\tilde{a}_{h}^{\delta}(v, v)=a_{h}^{\delta}(v, v) \geq \frac{1}{2}\|v\|_{E}=\frac{1}{2}\|v\|_{\tilde{E}} \quad \forall v \in W_{2}^{h} .
$$

Since the bilinear form $\tilde{a}_{h}^{\delta}(\cdot, \cdot)$ is no longer consistent, we employ Strang's Second Lemma to conclude

$$
\left\|u-u_{h}\right\|_{\tilde{E}} \leq C\left(\inf _{v \in W_{2}^{h}}\|u-v\|_{\tilde{E}}+\sup _{0 \neq v \in W_{2}^{h}} \frac{\left|\tilde{a}_{h}^{\delta}(u, v)-(f, v)\right|}{\|v\|_{\tilde{E}}}\right) .
$$


To bound the last term in (4.14), we write

$$
\begin{aligned}
(f, v)= & -\delta\left(\bar{\nabla} \square u, \nabla E_{h} v\right)-(\Delta u, v)+\left(f+\Delta u, v-E_{h} v\right) \\
= & \sum_{T \in \mathcal{T}_{h}}\left(\delta(\square u, \square v)_{T}+(\nabla u, \nabla v)_{T}-\delta\left\langle\square u, \partial_{\bar{n}} v\right\rangle_{\partial T}-\left\langle\partial_{n} u, v\right\rangle_{\partial T}\right. \\
& \left.\quad-\delta\left(\bar{\nabla} \square u, \nabla\left(E_{h} v-v\right)\right)_{T}\right)+\left(f+\Delta u, v-E_{h} v\right) \\
= & \tilde{a}_{h}^{\delta}(u, v)-\delta \sum_{T \in \mathcal{T}_{h}}\left(\nabla \square u, \bar{\nabla}\left(E_{h} v-v\right)\right)_{T}+\left(f+\Delta u, v-E_{h} v\right) .
\end{aligned}
$$

Bounding the last two terms in the expression above, we use Lemma 4.3.

$$
\begin{aligned}
\delta\left|\sum_{T \in \mathcal{T}_{h}}\left(\bar{\nabla} \square u, \nabla\left(E_{h} v-v\right)\right)_{T}\right| & \leq \delta C\|\nabla \square u\|_{L^{2}}\left(\sum_{e \in \mathcal{E}_{h}} h_{e}^{-1}\|[v]\|_{L^{2}(e)}^{2}\right)^{\frac{1}{2}} \\
& \leq \delta C h\|\nabla \square u\|_{L^{2}}\|v\|_{\tilde{E}}, \\
\left|\left(f+\Delta u, v-E_{h} v\right)\right| & \leq C\left(\|f\|_{L^{2}}+\|\Delta u\|_{L^{2}}\right)\left(\sum_{e \in \mathcal{E}_{h}} h_{e}\|[v]\|_{L^{2}(e)}^{2}\right)^{\frac{1}{2}} \\
& \leq C h^{2}\left(\|f\|_{L^{2}}+\|\Delta u\|_{L^{2}}\right)\|v\|_{\tilde{E}} .
\end{aligned}
$$

Thus by (2.3),

$$
\begin{aligned}
\sup _{v \in W_{2}^{h}} \frac{\left|\tilde{a}_{h}^{\delta}(u, v)-(f, v)\right|}{\|v\|_{\tilde{E}}} & \leq C h\left(\delta\|\nabla \square u\|_{L^{2}}+h\left(\|\Delta u\|_{L^{2}}+\|f\|_{L^{2}}\right)\right) \\
& \leq C M_{0} h(\sqrt{\delta}+h)\|f\|_{L^{2}} .
\end{aligned}
$$

Next, it is clear from the proof of Lemma 4.1 that

$$
\inf _{v \in W_{r}^{h}}\|u-v\|_{\tilde{E}} \leq C h(\sqrt{\gamma+\delta}+h)\|u\|_{H^{3}} .
$$

Combining (4.14)-(4.16), we obtain

$$
\left\|u-u_{h}\right\|_{\tilde{E}} \leq C h\left\{(\sqrt{\gamma+\delta}+h)\|u\|_{H^{3}}+M_{0}(\sqrt{\delta}+h)\|f\|_{L^{2}}\right\} .
$$

Remark 4.2. Using a standard duality argument ([3, 13]) and the regularity estimates (2.3), it is also possible to show the following $L^{2}$-error estimates when $u \in H^{4}(\Omega)$ :

$$
\begin{array}{ll}
\left\|u-u_{h}\right\|_{L^{2}} \leq C h^{\ell-1}(\sqrt{\gamma+\delta}+h)\|u\|_{H^{\ell}}, & r \geq 3, \\
\left\|u-u_{h}\right\|_{L^{2}} \leq C h^{2}(\sqrt{\gamma+\delta}+h)\|u\|_{H^{4}}, & r=2 .
\end{array}
$$

\section{NUMERICAL EXPERIMENTS}

In this section, we provide numerical experiments to gauge the efficiency of the finite element methods developed in the previous sections. We calculate the rate of convergence of $\left\|u-u_{h}\right\|$ for fixed $\delta$ in various norms and compare each computed rate with its theoretical estimate. 
Test 1. For this test, we solve (3.2) using the Morley-type nonconforming element $S_{2}^{h}$ defined in Section 3 with $\Omega=(0,1)^{2}$ and $\gamma=\delta$. We use the following data:

$$
\begin{aligned}
f= & -8 \pi^{4} \delta\left(\cos ^{2}(\pi x)-\sin ^{2}(\pi y)\right) \\
& -2 \pi^{2}\left\{\sin ^{2}(\pi y)\left(\cos ^{2}(\pi x)-\sin ^{2}(\pi x)\right)+\sin ^{2}(\pi x)\left(\cos ^{2}(\pi y)-\sin ^{2}(\pi y)\right)\right\},
\end{aligned}
$$

so that the exact solution is

$$
u=\sin ^{2}(\pi x) \sin ^{2}(\pi y) .
$$

We list the errors along with their estimated rates of convergence in Table 1 for $\delta$-values $10,1,10^{-2}, 10^{-3}$, and $10^{-4}$ and plot the errors in Figures 3 and 4 . The table indicates the following rates of convergence:

$$
\begin{array}{ll}
\left\|u-u_{h}\right\|_{M}=O(h), \quad & \left\|u-u_{h}\right\|_{2, h}=O(h), \\
\left\|u-u_{h}\right\|_{1, h}=O\left(h^{2}\right), & \left\|u-u_{h}\right\|_{L^{2}}=O\left(h^{2}\right) .
\end{array}
$$

Ignoring the insignificant $|\ln h|$ terms in (3.10)-(3.11), (3.15), these are the same rates of convergence established in Theorems 3.1 and 3.2. We also observe that as $\delta \rightarrow 0^{+}$, the error increases in the $L^{2}, H^{1}$, and $H^{2}$ norms which is expected by the definition of the constants in the error estimates (3.11) and (3.15).

Finally, we solve (3.2) but with $\gamma \equiv 0$ and $\delta=1$. Table 2 clearly shows that the method does not converge, this then indicates that the penalty terms in the method are essential.

Test 2. In this test, we compute the solution of the discontinuous Galerkin method (4.4) with $r=2$ and $\gamma=100 \delta$. We use the same domain and test functions as in Test 1 . We list the errors in Table 3 and plot the computed errors in Figures 5 and 6 for $\delta$-values $10,1,10^{-2}$, and $10^{-4}$. As expected, the rates of convergence depend on both the parameters $h$ and $\delta$. In fact, Theorem 4.2 and Remark 4.2 tell us that for $\sqrt{\delta}>>h, \gamma \approx \delta$,

$$
\begin{aligned}
& \left\|u-u_{h}\right\|_{\tilde{E}} \leq C h(\sqrt{\delta}+h)\left(\|u\|_{H^{3}}+\|f\|_{L^{2}}\right) \leq C h\left(\|u\|_{H^{3}}+\|f\|_{L^{2}}\right), \\
& \left\|u-u_{h}\right\|_{1, h} \leq C h(\sqrt{\delta}+h)\left(\|u\|_{H^{3}}+\|f\|_{L^{2}}\right) \leq C h\left(\|u\|_{H^{3}}+\|f\|_{L^{2}}\right), \\
& \left\|u-u_{h}\right\|_{L^{2}} \leq C h^{2}(\sqrt{\delta}+h)\|u\|_{H^{4}} \leq C h^{2}\|u\|_{H^{4}},
\end{aligned}
$$

where, as for $\sqrt{\delta} \leq h, \gamma \approx \delta$,

$$
\begin{aligned}
& \left\|u-u_{h}\right\|_{\tilde{E}} \leq C h(\sqrt{\delta}+h)\left(\|u\|_{H^{3}}+\|f\|_{L^{2}}\right) \leq C h^{2}\left(\|u\|_{H^{3}}+\|f\|_{L^{2}}\right), \\
& \left\|u-u_{h}\right\|_{1, h} \leq C h(\sqrt{\delta}+h)\left(\|u\|_{H^{3}}+\|f\|_{L^{2}}\right) \leq C h^{2}\left(\|u\|_{H^{3}}+\|f\|_{L^{2}}\right), \\
& \left\|u-u_{h}\right\|_{L^{2}} \leq C h^{2}(\sqrt{\delta}+h)\|u\|_{H^{4}} \leq C h^{3}\|u\|_{H^{4}} .
\end{aligned}
$$

As seen from Figure 5 , the computed error bounds agree with these theoretical error bounds. Finally, we note that $\left\|u-u_{h}\right\|_{2, h}$ appears not to converge unlike the previous test. However, additional numerical experiments (not listed here) indicate that $\left\|u-u_{h}\right\|_{2, h}$ converges with order $O\left(h^{2}\right)$ if cubic polynomials are used. 

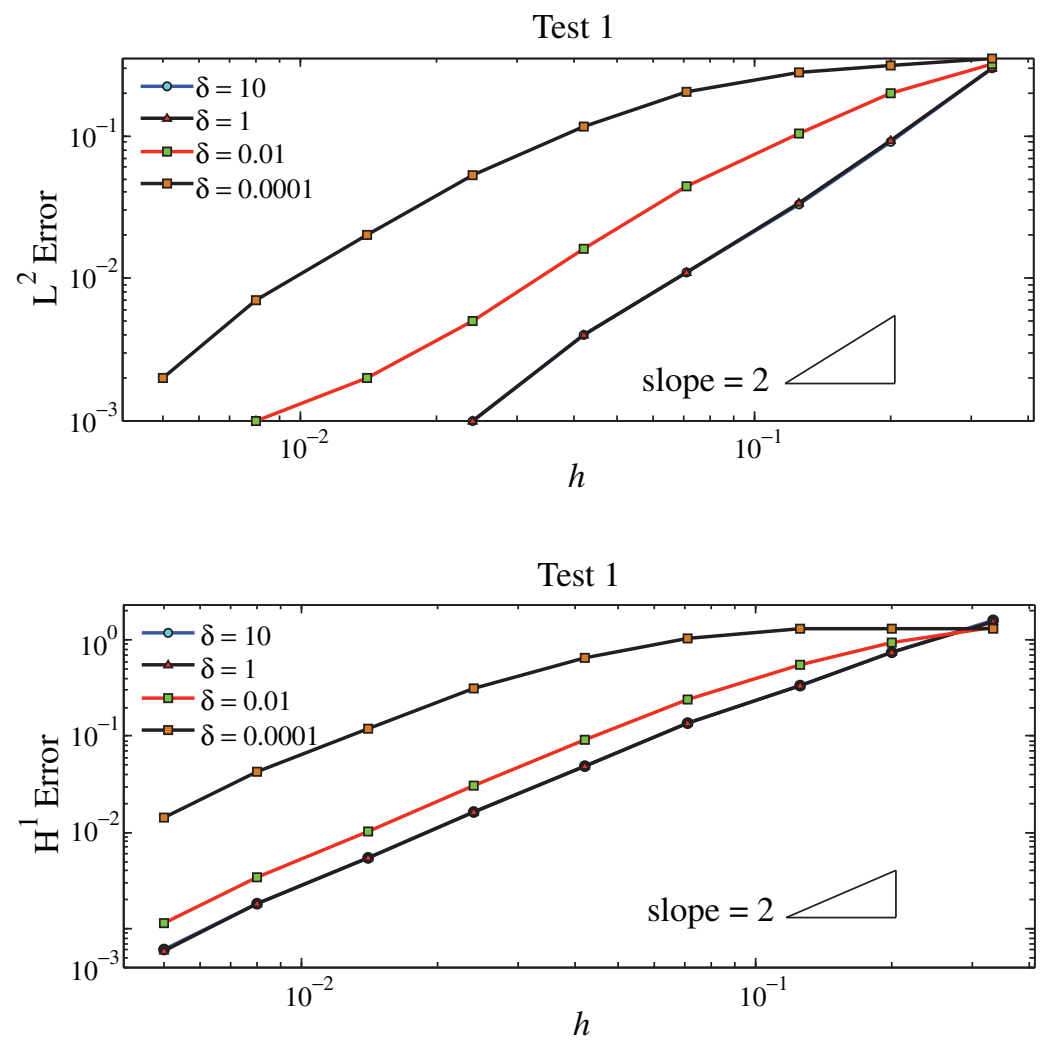

FiguRe 3. Test 1. $L^{2}$ norm (top) and $H^{1}$ norm (bottom) errors w.r.t. $h$ with $\delta=10,1,10^{-2}, 10^{-3}$, and $10^{-4}$. 
Test 1

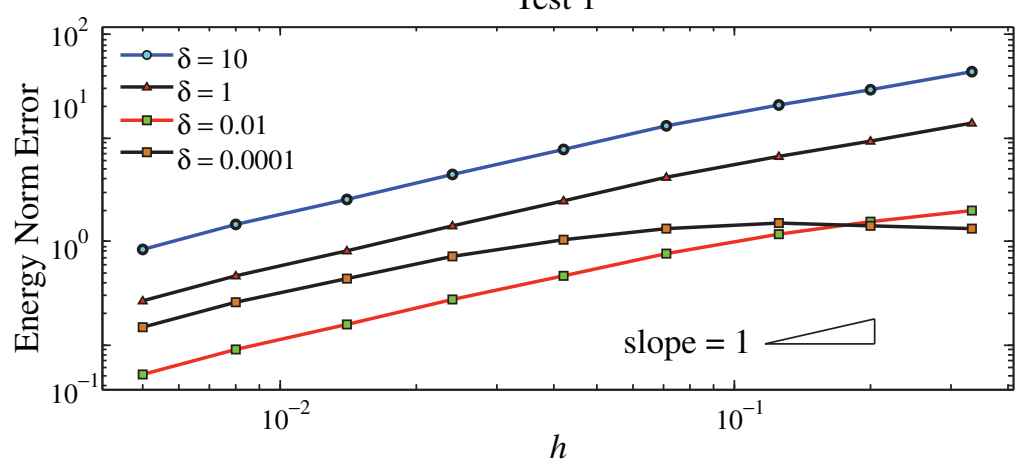

Test 1

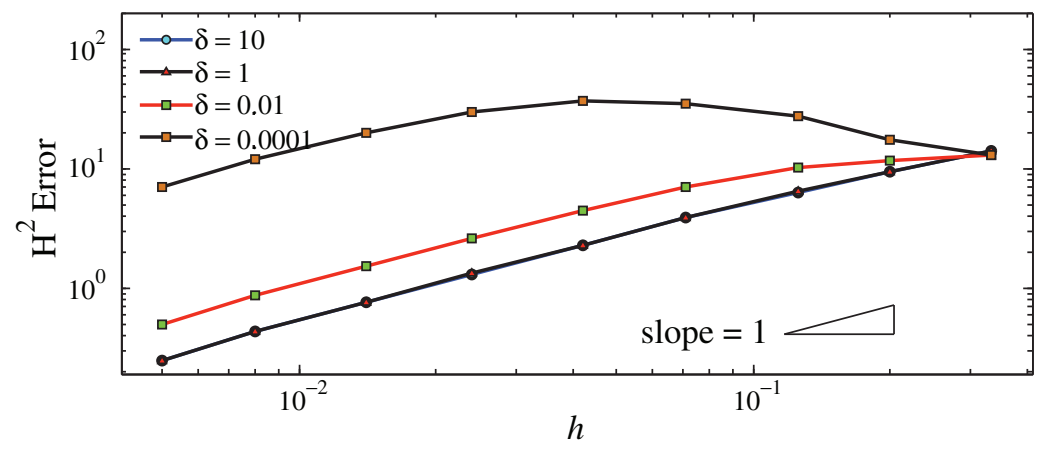

Figure 4. Test 1 . Energy norm (top) and $H^{2}$ norm (bottom) errors w.r.t. $h$ with $\delta=10,1,10^{-2}, 10^{-3}$ and $10^{-4}$. 
Test 2

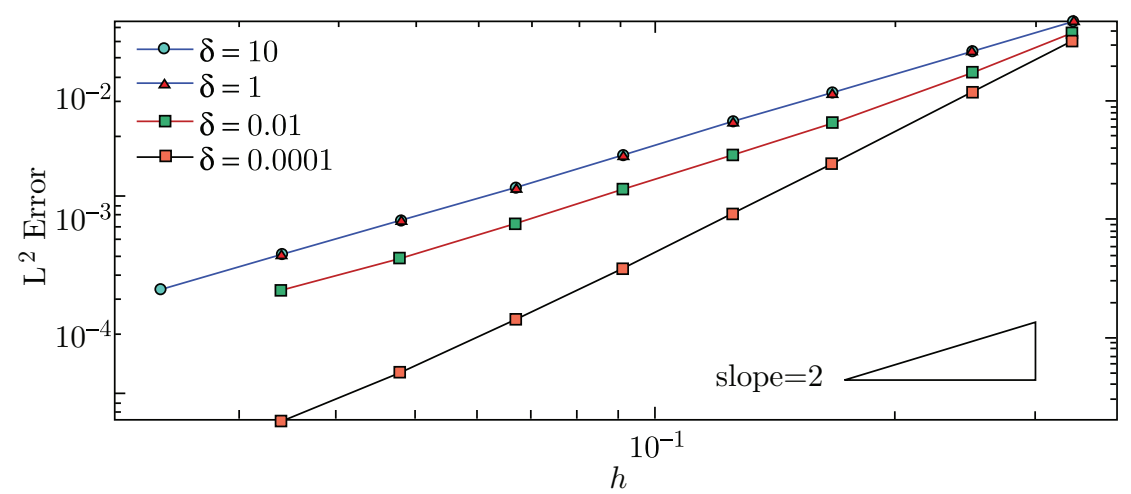

Test 2

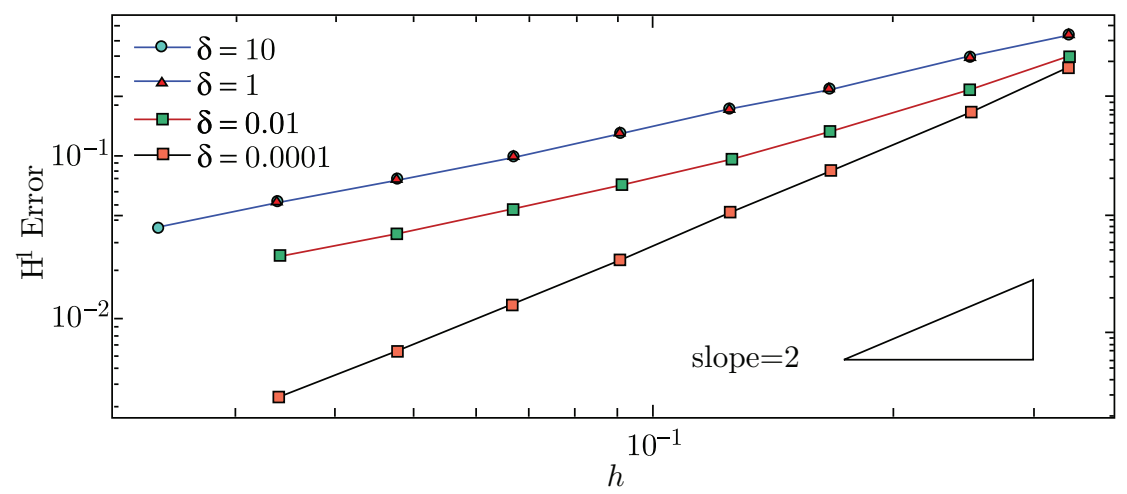

Figure 5. Test 2. $L^{2}$-norm (top) and $H^{1}$-norm (bottom), errors w.r.t. $h$ with $\delta=10,1,10^{-2}$ and $10^{-4}$. 
Test 2

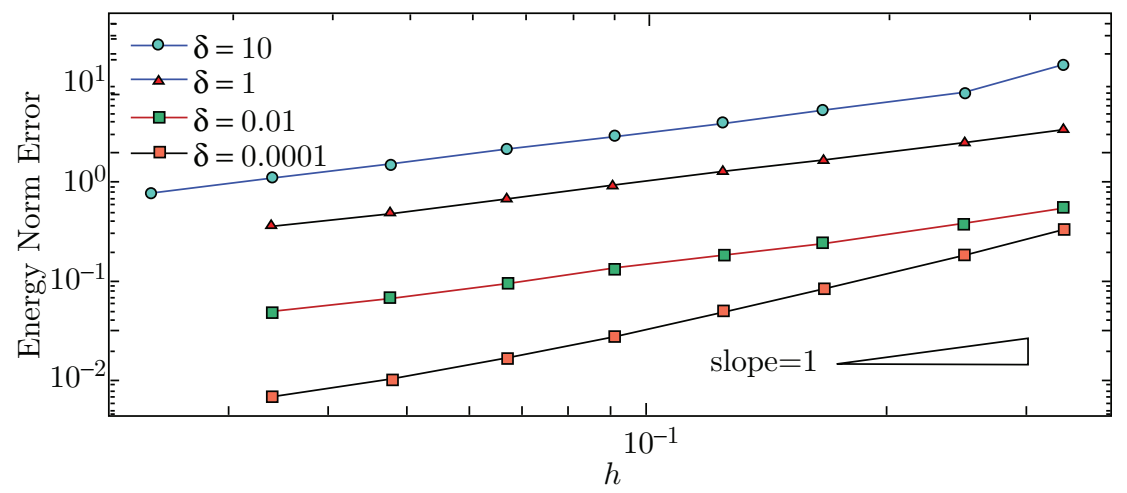

Test 2

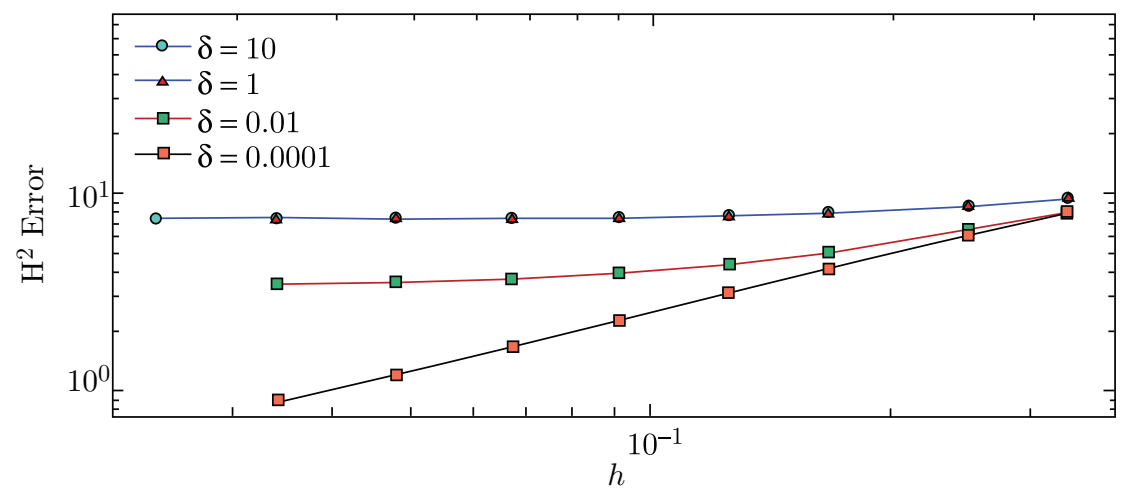

Figure 6. Test 2. Energy norm (top) and $H^{2}$-norm (bottom), errors w.r.t. $h$ with $\delta=10,1,10^{-2}$ and $10^{-4}$. 
TABLE 1. Test 1. Errors with estimated rates of convergence $(\gamma=\delta)$.

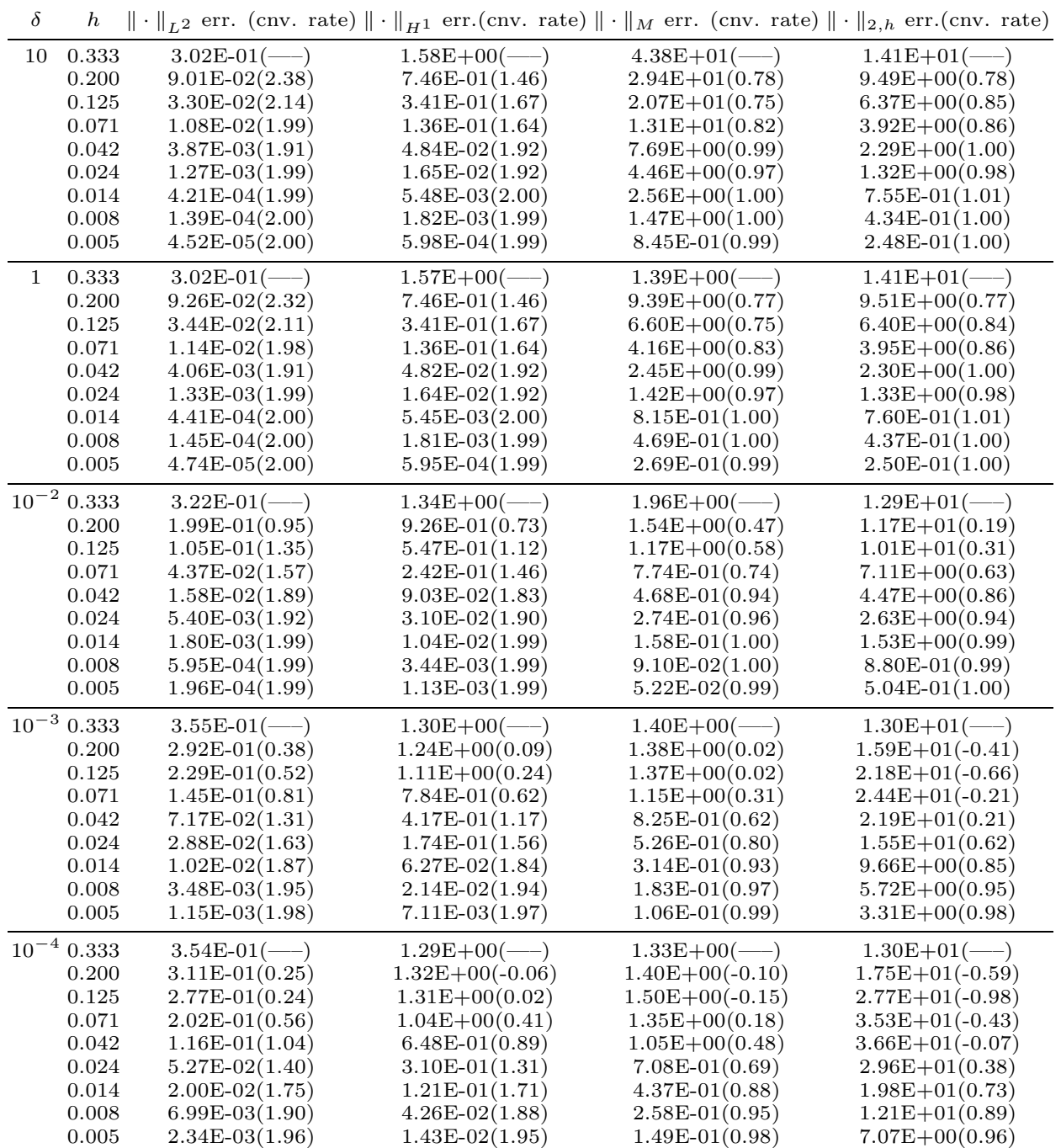

TABLE 2. Test 1 . Errors with $\delta=1$ and $\gamma \equiv 0$.

\begin{tabular}{cccrrr}
$\delta$ & $h$ & $\|\cdot\|_{L^{2}}$ err. & $\|\cdot\|_{H^{1}}$ err. & $\|\cdot\|_{M}$ err. & $\|\cdot\|_{2, h}$ err. \\
\hline 1 & 0.333 & $1.78 \mathrm{e}+01$ & $6.46 \mathrm{e}+01$ & $6.51 \mathrm{e}+01$ & $4.57 \mathrm{e}+02$ \\
& 0.250 & $1.68 \mathrm{e}+01$ & $6.21 \mathrm{e}+01$ & $6.24 \mathrm{e}+01$ & $6.64 \mathrm{e}+02$ \\
& 0.167 & $1.65 \mathrm{e}+01$ & $6.46 \mathrm{e}+01$ & $6.49 \mathrm{e}+01$ & $1.12 \mathrm{e}+03$ \\
& 0.125 & $1.72 \mathrm{e}+01$ & $6.74 \mathrm{e}+01$ & $6.78 \mathrm{e}+01$ & $1.95 \mathrm{e}+03$ \\
& 0.091 & $1.71 \mathrm{e}+01$ & $6.77 \mathrm{e}+01$ & $6.81 \mathrm{e}+01$ & $3.40 \mathrm{e}+03$ \\
& 0.067 & $1.73 \mathrm{e}+01$ & $6.82 \mathrm{e}+01$ & $6.87 \mathrm{e}+01$ & $5.93 \mathrm{e}+03$ \\
& 0.048 & $1.72 \mathrm{e}+01$ & $6.82 \mathrm{e}+01$ & $6.87 \mathrm{e}+01$ & $1.03 \mathrm{e}+04$ \\
& 0.034 & $1.72 \mathrm{e}+01$ & $6.82 \mathrm{e}+01$ & $6.87 \mathrm{e}+01$ & $1.80 \mathrm{e}+04$ \\
& 0.024 & $1.73 \mathrm{e}+01$ & $6.83 \mathrm{e}+01$ & $6.88 \mathrm{e}+01$ & $3.14 \mathrm{e}+04$
\end{tabular}


TABLE 3. Test 2. Errors with estimated rates of convergence

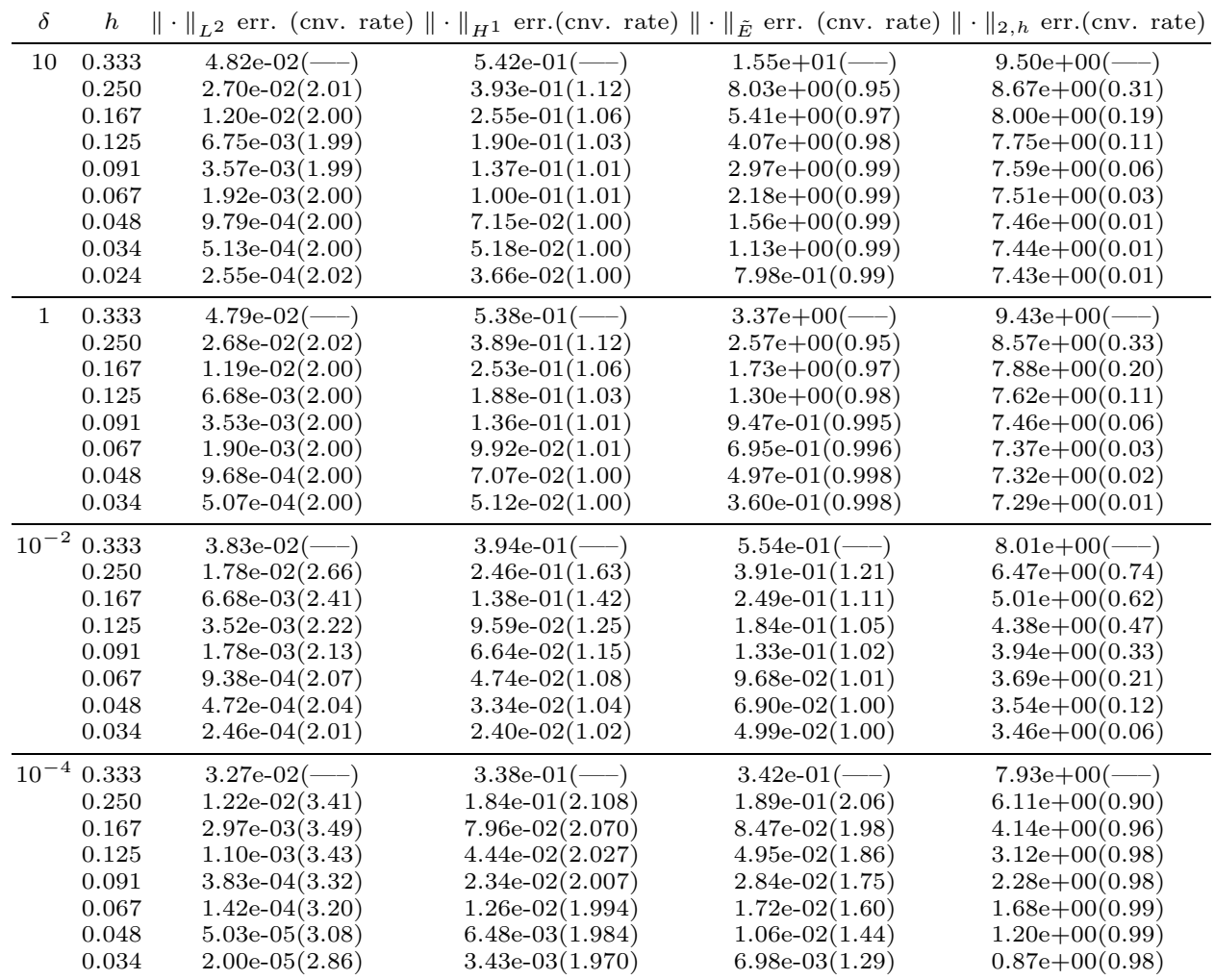

\section{ACKNOWLEDGMENTS}

The work of both authors was partially supported by the NSF grant DMS0710831. The authors would like to thank Professor Qiang Du of Penn State University for bringing the bi-wave problem to their attention and for providing the relevant references on $d$-wave superconductors.

The authors would like to thank the anonymous referees for their critical questions and valuable suggestions which not only helped the authors to avoid some errors in the early version of the paper, but also led to significant improvements on the results of Section 3.3 .

\section{REFERENCES}

[1] D. Arnold, An interior penalty finite element method with discontinuous elements, SIAM J. Numer. Anal., 19:742-760, 1982. MR664882 (83f:65173)

[2] D. Arnold, F. Brezzi, B. Cockburn, and L. Marini, Unified analysis for discontinuous Galerkin methods for elliptic problems, SIAM J. Numer. Anal., 39(5):1749-1779, 2002. MR1885715 (2002k:65183)

[3] G. A. Baker, Finite element methods for elliptic equations using nonconforming elements, Math. Comp. 31(137):45-59, 1977. MR0431742 (55:4737)

[4] D. G. Bourgin and R. Duffin, The Dirichlet problem for the vibrating string equation, Bull. Amer. Math. Soc., 45:851-858, 1939. MR0000729 (1:120f) 
[5] S. C. Brenner, Discrete Sobolev and Poincaré inequalities for piecewise polynomial functions, Electr. Trans. Numer. Anal., 18:42-48, 2004. MR2083293 (2005k:65239)

[6] S. C. Brenner and L. R. Scott, The Mathematical Theory of Finite Element Methods, third edition, Springer, 2008. MR2373954 (2008m:65001)

[7] P. G. Ciarlet, The Finite Element Method for Elliptic Problems. North-Holland, Amsterdam, 1978. MR0520174 (58:25001)

[8] Q. Du, Studies of Ginzburg-Landau model for d-wave superconductors, SIAM J. Appl. Math, 59(4):1225-1250, 1999. MR 1686712 (2000b:82045)

[9] L. C. Evans, Partial Differential Equations, volume 19 of Graduate Studies in Mathematics, American Mathematical Society, Providence, RI, 1998. MR.1625845 (99e:35001)

[10] J. Douglas, Jr. and T. Dupont, Interior penalty procedures for elliptic and parabolic Galerkin methods, Lecture Notes In Physics 58, Springer-Verlag, Berlin, 1976. MR0440955 (55:13823)

[11] D. L. Feder and C. Kallin, Microscopic derivation of the Ginzburg-Landau equations for a d-wave superconductor, Phys. Rev. B, 55:559-574, 1997.

[12] X, Feng and O. A. Karakashian, Fully discrete dynamic mesh discontinuous Galerkin methods for the Cahn-Hilliard equation of phase transition, Math. Comp. 76:1093-1117, 2007. MR2299767 (2008a:74048)

[13] X. Feng and M. Neilan, Finite element methods for a bi-wave equation modeling d-wave superconductors, J. Comput. Math., 28(3):331-353, 2010.

[14] V. L. Ginzburg and L. D. Landau, On the theory of superconductivity, Zh. Eksper. Teoret. Fiz. 20:1064-1082, 1950 (in Russian), in: L.D. Landau, and I.D. ter Haar (Eds.), Man of Physics, Pergamon, Oxford, 1965, pp. 138-167 (in English).

[15] O. A. Karakashian and F. Pascal, A posteriori error estimates for a discontinuous Galerkin approximation of second-order elliptic problems, SIAM J. Numer. Anal., 41(6):2374-2399, 2003. MR2034620 (2005d:65192)

[16] A. Lasis and E. Süli, Poincaré-type inequalities for broken Sobolev spaces, Oxford University Computing Laboratory, Numerical Analysis Technical Report, 03(10), 2003.

[17] L. Morley, The triangular equilibrium element in the solution of plate bending problems, Aero. Quart. 19, 149-169, 1968.

[18] Y. Ren, J.-H. Xu, and C. S. Ting, Ginzburg-Landau equations for mixed $s+d$ symmetry superconductors, Phys. Rev. B, 53:2249-2252, 1996.

[19] B. Rivière, Discontinuous Galerkin Methods for Solving Elliptic and Parabolic Equations, SIAM, 2008. MR2431403 (2009f:65260)

[20] Z. C. Shi, On the error estimate of Morley element. Numer. Math. Sinica, 12(2):113-118, 1990. MR1070298(91i:65182)

[21] M. Tinkham, Introduction to Superconductivity, Second Edition, Dover Publications, 2004.

[22] M. Wang and J. Xu, The Morley element for fourth-order elliptic equations in any dimension, Numer. Math. 103:155-169, 2006. MR2207619 (2006i:65205)

[23] M. F. Wheeler, An elliptic collocation-finite element method with interior penalties., SIAM J. Numer. Anal., (15), 1978, pp. 152-161. MR0471383 (57:11117)

[24] J.-H. Xu, Y. Ren, and C. S. Ting, Ginzburg-Landau equations for a d-wave superconductor with nonmagnetic impurities, Phys. Rev. B, 53(18):12481-12495, 1996. 37996

Department of Mathematics, The University of Tennessee, Knoxville, Tennessee

E-mail address: xfeng@math.utk.edu

Center for Computation and Technology and Department of Mathematics, Louisiana State University, Baton Rouge, Louisiana 70808

E-mail address: neilan@math.lsu.edu 